\title{
AN INDEX THEORY FOR QUANTUM DYNAMICAL SEMIGROUPS
}

\author{
B. V. RAJARAMA BHAT
}

\begin{abstract}
W. Arveson showed a way of associating continuous tensor product systems of Hilbert spaces with endomorphism semigroups of type I factors. We do the same for general quantum dynamical semigroups through a dilation procedure. The product system so obtained is the index and its dimension is a numerical invariant for the original semigroup.
\end{abstract}

\section{INTRODUCTION}

Powers [Po1] initiated an index theory for $E_{0}$-semigroups. By definition $E_{0^{-}}$ semigroups are continuous (weak operator topology) semigroups of normal $*$-endomorphisms of the algebra of all bounded operators on a separable infinite dimensional Hilbert space. The theory has seen considerable progress with contributions of Powers and his co-authors ([Po1],[Po2], [PR], [PP]), Arveson [Ar1, Ar2, Ar3, Ar4, Ar5, Ar6, Ar7], Price [Pr], and others. It is the work of Arveson (especially [Ar1]) which concerns us most here. He is able to associate a product system with every $E_{0}$-semigroup in a canonical manner. By a product system we mean a family of Hilbert spaces $\left\{\mathcal{E}_{t}, t>0\right\}$, satisfying

$$
\mathcal{E}_{s+t} \cong \mathcal{E}_{s} \otimes \mathcal{E}_{t}, \quad s, t>0,
$$

in an 'associative' way with some additional measurability conditions. Earlier such systems played a decisive role in the famous work of Araki and Woods [AW]. The paradigm examples are got through symmetric Fock spaces (see $[\mathrm{Gu}],[\mathrm{PaS}]$ ). However it is known that there are $E_{0}$-semigroups $[\mathrm{Po} 2]$ which lead to more intricate product systems. The index of an $E_{0}$-semigroup is defined to be the isomorphism class of the associated product system. This way the index becomes an invariant for the semigroup. With some further investigation [Ar1] it is possible to obtain a numerical invariant by defining a 'dimension' for the product system. Moreover it is possible to classify $E_{0}$-semigroups more or less completely using product systems [Ar6].

Here the scope of this index theory has been enhanced considerably to admit more general semigroups. Fix a complex separable Hilbert space $\mathcal{H}_{0}$ (It could even be finite dimensional.) Let $T=\left\{T_{t}, t \geq 0\right\}$ be a semigroup of unital, normal

Received by the editors May 27, 1994.

1991 Mathematics Subject Classification. Primary 46L57, 81S25, 46L55.

Key words and phrases. Completely positive maps, semigroups, Markov dilations, continuous tensor products.

This research was supported by a fellowship from INDAM (ITALY). 
completely positive maps on $\mathcal{B}\left(\mathcal{H}_{0}\right)$. With every such semigroup $T$ we are able to associate a family $\mathcal{P}=\left\{\mathcal{P}_{t}\right\}$ of Hilbert spaces satisfying

$$
\mathcal{P}_{s+t} \cong \mathcal{P}_{s} \otimes \mathcal{P}_{t}, \quad s, t>0 .
$$

The separability of these Hilbert spaces can be ensured along with fulfillment of other technical conditions to have a product system in the sense of Arveson under a mild regularity assumption of path-continuity (see Definition 5.1, Proposition 5.2, 5.3 ) on the semigroup.

The constructions are based on weak Markov dilations ([Bh], [BP2]) of quantum dynamical semigroups (semigroups of contractive completely positive maps on a $C^{*}$-algebra). This theory allows us to construct a family $j=\left\{j_{t}\right\}$ of (non-unital) *-homomorphisms, $j_{t}: \mathcal{B}\left(\mathcal{H}_{0}\right) \rightarrow \mathcal{B}(\mathcal{H})$, for some Hilbert space $\mathcal{H}$ containing $\mathcal{H}_{0}$. We see that up to unitary isomorphisms the range of $j_{t}(I)$ (denoted by $\mathcal{H}_{t]}$ ) splits as $\mathcal{H}_{0} \otimes \mathcal{P}_{t}$, with $\left\{\mathcal{P}_{t}\right\}$ satisfying (1.1). Finally using the family $\left\{\mathcal{P}_{t}\right\}$ we are able to 'dilate' $T$ to a semigroup $\theta$ of $*$-endomorphisms of $\mathcal{B}(\mathcal{H})$ (Theorem 4.7). This semigroup is uniquely determined up to unitary isomorphisms. We define the index of $T$ by simply taking it as index of $\theta$. It is seen to be anti-isomorphic to the product system $\mathcal{P}$ obtained earlier. Here we emphasize that the index is important for us not only because it gives an invariant for the semigroup but also it provides a factorization of the dilation.

If $T$ happens to be a semigroup of endomorphisms then its dilation is itself. That is, we have $\mathcal{H}=\mathcal{H}_{0}$, and $\theta=j=T$. Here the theory matches with Arveson's work, except for the method of constructing product systems. A notable improvement being that we obtain all the constituent Hilbert spaces of the product system as subspaces of a single space, the dilation space. Moreover the orthogonal projections onto these subspaces form a strongly continuous family. Whereas in [Ar1] the necessary Hilbert spaces are obtained by imposing a somewhat artificial innerproduct on some subspaces of $\mathcal{B}(\mathcal{H})$. (So in that setting there is no compatible inner-product between vectors belonging to two different subspaces.)

The product system $\mathcal{P}$ describes a multiplicative structure of the dilation. Roughly speaking there is also an additive structure when we deal with general quantum dynamical semigroups. Actually, we obtain another family of Hilbert spaces $\left\{\mathcal{N}_{t}\right\}$, now decomposing $\mathcal{H}_{t]}$ as $\mathcal{H}_{0} \oplus \mathcal{N}_{t}$, and satisfying

$$
\mathcal{N}_{s+t} \cong \mathcal{N}_{s} \oplus\left(\mathcal{N}_{t} \otimes \mathcal{P}_{s}\right)
$$

A detailed study of such systems is yet to be undertaken.

The associated product systems become trivial (i.e., $\mathcal{P}_{s} \cong \mathbb{C}$ ) and hence (1.2) becomes completely additive when we deal with quantum dynamical semigroups implemented by semigroups of contractions. At the other extreme only the product structure is non-trivial (i.e., $\mathcal{N}_{t} \cong\{0\}$ ) for semigroups of endomorphisms.

The paper has been ordered as follows. We begin with the definition of quantum dynamical semigroups and give a brief summary of the results we are going to need from the dilation theory in Section 2. Discrete time quantum dynamical semigroups are dealt with in Section 3. Here dilations have been constructed quite explicitly. Naturally enough we obtain 'discrete product systems'.

In subsequent sections we consider conservative (unital), normal, quantum dynamical semigroups in continuous time. The theory is in complete parallel with the discrete time version. The basic constructions of spaces $\mathcal{P}, \mathcal{N}$ with associated 
unitary maps are in Section 4. Main results being factorization of $j$, and the existence and uniqueness of the endomorphism semigroup $\theta$. (Theorems 4.5-4.8). In a sense, we have a semigroup version of Stinespring's theorem. As a simple corrollary to the dilation theory it is seen that if a quantum dynamical semigroup on $\mathcal{B}\left(\mathcal{H}_{0}\right)$ is an endomorphism for some positive time point then it is a semigroup of endomorphisms.

In Section 5 we begin with explaining the notion of path-continuity which guarantees the separability of the dilation space. This point onwards, path-continuity is an added assumption on the semigroup. After briefly recalling the definition of product systems we see that our construction does lead to such a family of Hilbert spaces. This is linked up with Arveson's construction of product systems and associated index theory in the final section. Some functorial properties of the index such as invariance under cocycle conjugacy can be seen. Units of a product system are families of vectors with a special multiplicative property, and form the basis for Arveson's study of product systems. Theorem 6.4 explicitly provides units, and in particular shows that the numerical index is positive for semigroups with pure invariant states.

\section{MARKOV Dilations OF QUANTUM DYNAMICAL SEMIGROUPS}

Quantum dynamical semigroups appear naturally in various physical contexts ([Da1], [Da2], [AL]). And one studies them either by looking at the structure of the generators ([GKS], [Li], [Da4]) or by constructing suitable dilations ([EL], [HP]). Quantum dynamical semigroups are non-commutative versions of Markov semigroups and their dilations to homomorphisms can be interpreted as construction of non-commutative or quantum Markov processes. Accardi, Friegerio and Lewis [AFL] formulated this idea in a precise mathematical format and obtained a reconstruction theorem. We will not be needing this general theory here. All our constructions will be based on the theory of weak Markov dilations of quantum dynamical semigroups as in $[\mathrm{Bh}]$ and $[\mathrm{BP} 2]$. (See [Em], [Sa] and [ViS] for some earlier work.)

In the sequel $\mathbb{T}_{+}$will denote either the additive semigroup $\mathbb{Z}_{+}$of nonnegative integers or $\mathbb{R}_{+}$of nonnegative real numbers.

Definition 2.1. Let $\mathcal{A}_{0}$ be a unital $C^{*}$ algebra. A family of linear maps $T=\left\{T_{t}\right.$ : $\left.t \in \mathbb{T}_{+}\right\}$, of $\mathcal{A}_{0}$ into itself is called a quantum dynamical semigroup if the following are satisfied:

(i) $T_{t}$ is completely positive for every $t \in \mathbb{T}_{+}$;

(ii) $T_{s}\left(T_{t}(X)\right)=T_{s+t}(X)$ for all $X \in \mathcal{A}_{0}, s, t \in \mathbb{T}_{+}$;

(iii) $T_{0}(X)=X$ for all $X \in \mathcal{A}_{0}$;

(iv) $T_{t}(I) \leq I$ for all $t \in \mathbb{T}_{+}$.

The semigroup is called conservative or unital if $T_{t}(I)=I$ for every $t$.

As of now there is no continuity restriction on the semigroup in the variable $t$. In discrete time (i.e., $\mathbb{T}_{+}=\mathbb{Z}_{+}$) the dynamical semigroup consists of $\left\{I, T, T^{2}, \cdots\right\}$ for a single contractive completely positive map $T: \mathcal{A}_{0} \rightarrow \mathcal{A}_{0}$.

We motivate the dilation theory needed here through Sz. Nagy dilations of contractions. Let $R=\left\{R(t), t \in \mathbb{T}_{+}\right\}$be a contraction semigroup on a Hilbert space $\mathcal{H}_{0}$. Then $\left\{T_{t}\right\}$ defined by $T_{t}(X)=R(t) X R(t)^{*}$ is a quantum dynamical semigroup on $\mathcal{B}\left(\mathcal{H}_{0}\right)$. Now by [SzF] there exists a Hilbert space $\mathcal{H}$ containing $\mathcal{H}_{0}$ 
with a semigroup of isometries $V$, such that

$$
R(t)=\left.P V(t)\right|_{\mathcal{H}_{0}}
$$

where $P$ is the projection of $\mathcal{H}$ onto $\mathcal{H}_{0}$. Let $F(t)$ be the projection onto the closed linear span of $\left\{V(s) u: 0 \leq s \leq t, u \in \mathcal{H}_{0}\right\}$. Define $j_{t}: \mathcal{B}\left(\mathcal{H}_{0}\right) \rightarrow \mathcal{B}(\mathcal{H})$ by

$$
j_{t}(X)=V(t) P X P V(t)^{*} \text { for } t \in \mathbb{T}_{+} \text {and } X \in \mathcal{B}\left(\mathcal{H}_{0}\right) \text {. }
$$

Then $j=\left\{j_{t}, t \in \mathbb{T}_{+}\right\}$, is a family of representations of $\mathcal{B}\left(\mathcal{H}_{0}\right)$ satisfying $j_{t}(I) \leq$ $F(t)$, and $F(s) j_{t}(I) F(s)=j_{s}\left(T_{t-s}(X)\right)$, for $s \leq t$. The idea is to construct representations having similar properties with respect to a given quantum dynamical semigroup.

We will be dealing with only a special subclass of Markov dilations called subordinate weak Markov flows. For a more general definition of Markov flows see ([BP2], [BP3]).

Definition 2.2. Let $\mathcal{A}_{0}$ be a unital $C^{*}$ algebra of operators on a Hilbert space $\mathcal{H}_{0}$ and let $T=\left\{T_{t}, t \in \mathbb{T}_{+}\right\}$be a quantum dynamical semigroup on $\mathcal{A}_{0}$. A triple $(\mathcal{H}, F, j)$ is called a subordinate weak Markov flow with expectation semigroup $\left\{T_{t}\right\}$ if $\mathcal{H}$ is a Hilbert space containing $\mathcal{H}_{0}$ as a subspace, $F=F(t)$ is a non-decreasing family of orthogonal projections on $\mathcal{H}$ with $F(0)$ having range $\mathcal{H}_{0}$, and $j=\left\{j_{t}\right\}$ is a family of $*$-homomorphisms from $\mathcal{A}_{0}$ into $\mathcal{B}(\mathcal{H})$ satisfying the following:

(i) $j_{0}(X)=X F(0)$;

(ii) $F(s) j_{t}(X) F(s)=j_{s}\left(T_{t-s}(X)\right)$ for all $0 \leq s \leq t<\infty, X \in \mathcal{A}_{0}$.

The flow is called minimal if $\left\{j_{t_{1}}\left(X_{1}\right) \cdots j_{t_{n}}\left(X_{n}\right) u: t_{1}, \ldots, t_{n} \in \mathbb{T}_{+}, X_{1}, \ldots, X_{n}\right.$ $\in \mathcal{A}_{0}, u \in \mathcal{H}_{0}$ and $\left.n=1,2, \ldots\right\}$ is total (linear combinations form a dense set) in $\mathcal{H}$.

Note that (ii) implies that $j_{t}(I) \leq F(I)$; for this reason we say the flow is subordinate to $F$ and this in particular means that $j_{t}$ leaves the range of $F(t)$ invariant for every $t$. If $j_{t}(I)=F(t)$ for all $t$ then the flow is said to be conservative. We will not be needing any probabilistic ideas in the sequel, but we would like to point out that $F$ is to be thought of as a filtration and the map $Z \rightarrow F(t) Z F(t)$ occurring in (ii) as conditional expectation. So (ii) is essentially the Markov property of the flow.

Theorem 2.3. Given a quantum dynamical semigroup $T$ (as in Definition 2.2) up to unitary isomorphisms there is a unique minimal Markov flow $(\mathcal{H}, F, j)$ having $T$ as its expectation semigroup. Moreover $j$ is conservative if and only if $T$ is.

Proof. See [BP2] for the original proof. [Bh] has somewhat more direct approach. The central idea being the GNS construction as in the standard proof of Stinespring's theorem.

Throughout this article $(\mathcal{H}, F, j)$ guaranteed by Theorem 2.3 is the only flow we consider and we simply call it the minimal dilation of $T$. It is the Sz. Nagy dilation when $T$ is implemented by a semigroup of contractions on the Hilbert space (as explained before) and it is essentially the Kolmogorov construction of Markov processes when the algebra is abelian. Here we fix our notation and list some properties of this dilation for future reference.

The Hilbert space $\mathcal{H}_{0}$ (where the algebra $\mathcal{A}_{0}$ is acting) may be called the initial space and $\mathcal{H}$ the final space or the dilation space. The range of $F(t)$ is 'the Hilbert space up to time $t^{\prime}$ and is denoted by $\mathcal{H}_{t]}$. Note that $\mathcal{H}_{0]}$ is the same as $\mathcal{H}_{0}$. By 
convention $\mathcal{H}_{\infty}$ is $\mathcal{H}$. For any three time points $a, b, c$ in $\mathbb{T}_{+}$with $b \geq a, c$ and $X, Y, Z$ in $\mathcal{A}_{0}$, we have a 'reduction algorithm' (Proposition 3.2 of $[\mathrm{Bh}]$ ):

$$
j_{a}(X) j_{b}(Y) j_{c}(Z)= \begin{cases}j_{a}\left(X T_{b-a}(Y)\right) j_{c}(Z) & \text { if } c \leq a \leq b, \\ j_{a}(X) j_{c}\left(T_{b-c}(Y) Z\right) & \text { if } a \leq c \leq b, \\ j_{a}\left(X T_{b-a}(Y) Z\right) & \text { if } a=c \text { and } a \leq b .\end{cases}
$$

For any finite sequence $\underline{r}=\left(r_{1}, \ldots, r_{n}\right)$ in $\mathbb{T}_{+}$and $\underline{Y}=\left(Y_{1}, \ldots, Y_{n}\right)$ in $\mathcal{A}_{0}$ (of length $n)$ write $j(\underline{r}, \underline{Y})=j\left(r_{1}, r_{2}, \ldots, r_{n}, Y_{1}, \ldots, Y_{n}\right)=j_{r_{1}}\left(Y_{1}\right) j_{r_{2}}\left(Y_{2}\right) \ldots j_{r_{n}}\left(Y_{n}\right)$. If for some index $k$ in $\{2, \ldots, n-1\}, r_{k} \geq r_{k-1}, r_{k+1}$, then the algorithm can be applied at the triple $\left(r_{k-1}, r_{k}, r_{k+1}\right)$ to reduce the length of $\underline{r}, \underline{Y}$ in $j(\underline{r}, \underline{Y})$. Repeated application of the algorithm and (i) of Definition 2.2 to $F(0) j(\underline{r}, \underline{Y}) F(0)$ (recall $\left.F(0)=j_{0}(I)\right)$ yields $\epsilon(\underline{r}, \underline{Y}) \in \mathcal{A}_{0}$ such that $F(0) j(\underline{r}, \underline{Y}) F(0)=\epsilon(\underline{r}, \underline{Y}) F(0)$. Note that $\epsilon(\underline{r}, \underline{Y})$ is uniquely determined as $\langle u, \epsilon(\underline{r}, \underline{Y}) v\rangle=\langle u, j(\underline{r}, \underline{Y}) v\rangle$ for all $u, v \in \mathcal{H}_{0}$. The mapping $\epsilon$ satisfies

$$
\text { (i) } \epsilon(\underline{r}+s, \underline{Y})=T_{s}(\epsilon(\underline{r}, \underline{Y})) \text {; }
$$

$$
\text { (ii) } F(s) j(\underline{r}+s, \underline{Y}) F(s)=j_{s}(\epsilon(\underline{r}, \underline{Y})) \text {, }
$$

where $\underline{r}+s=\left(r_{1}+s, r_{2}+s, \ldots, r_{n}+s\right)$.

If $\underline{r}$ has some monotonicity property then one can chase the algorithm to get an explicit formula for $\epsilon(\underline{r}, \underline{Y})$. We find it useful to record a special case. Let $\mathcal{D}, \mathcal{D}_{t]}$ be the sets defined by

$$
\mathcal{D}=\left\{(\underline{r}, \underline{Y}, u): u \in \mathcal{H}_{0}, \underline{r}=\left(r_{1}, \ldots, r_{n}\right), r_{1}>r_{2}>\cdots>r_{n} \geq 0 \text { in } \mathbb{T}_{+},\right.
$$

$Y_{1}, \ldots Y_{n} \in \mathcal{A}_{0}$, for some $\left.n \geq 1\right\}$,

$$
\mathcal{D}_{t]}=\left\{(\underline{r}, \underline{Y}, u) \in \mathcal{D}: t \geq r_{1}>r_{2}>\cdots>r_{n} \geq 0\right\} .
$$

Then it follows from the construction in $[\mathrm{BP} 2]$ that $\left\{j(\underline{r}, \underline{Y}) u:(\underline{r}, \underline{Y}, u) \in \mathcal{D}_{t]}\right\}$ spans $\mathcal{H}_{t]}$ and

$$
\begin{aligned}
& \left\langle j(\underline{r}, \underline{Y}) u, j_{t}(X) j(\underline{r}, \underline{Z}) v\right\rangle \\
& \quad=\left\langle u, T_{r_{n}}\left(Y_{n}^{*} T_{r_{n-1}-r_{n}}\left(Y_{n-1}^{*} \cdots Y_{2}^{*} T_{r_{1}-r_{2}}\left(Y_{1}^{*} T_{t-r_{1}}(X) Z_{1}\right) Z_{2} \cdots Z_{n-1}\right) Z_{n}\right) v\right\rangle .
\end{aligned}
$$

for $X \in \mathcal{A}_{0},(\underline{r}, \underline{Y}, u),(\underline{r}, \underline{Z}, v) \in \mathcal{D}_{t]}$. Note that we have taken same time sequence $\underline{r}$ along with both $\underline{Y}$ and $\underline{Z}$. This is no restriction for the following reason. Consider $(\underline{r}, \underline{Y}, u) \in \mathcal{D}$. Then for any $\underline{s}=\left(s_{1}, \ldots, s_{p}\right)$ with $s_{1}>s_{2}>\cdots, s_{p} \geq 0$ in $\mathbb{T}_{+}$, we write $\underline{s} \geq \underline{r}$ if $\left\{s_{1}, \ldots, s_{p}\right\} \supseteq\left\{r_{1}, \ldots, r_{n}\right\}$ and in such a case define $(\underline{s}, \underline{\tilde{Y}}, u)$ by putting

$$
\tilde{Y}_{j}=\left\{\begin{array}{cl}
Y_{i} & \text { if } s_{j}=r_{i} \text { for some } i \\
I & \text { otherwise }
\end{array}\right.
$$

i.e., we extend $\underline{Y}$ by inserting identity at the extra time points. Then it follows from the reduction algorithm or otherwise that for conservative minimal flows,

$$
j(\underline{s}, \underline{\tilde{Y}}) u=j(\underline{r}, \underline{Y}) u .
$$

The same equality holds for nonconservative flows too under the added restriction $s_{1} \leq r_{1}$. Finally, we note the following technical result.

Proposition 2.4. Suppose $\mathcal{A}_{0}$ is a von Neumann subalgebra of $\mathcal{B}\left(\mathcal{H}_{0}\right)$ and $T_{t}$ is normal for $t \geq 0$. Then the homomorphisms $j_{t}$ are normal for every $t$. 
Proof. Suppose $X_{\alpha}$ is a net of positive elements of $\mathcal{A}_{0}$ monotonically increasing to $X$. Clearly $j_{t}\left(X_{\alpha}\right)$ is increasing. We need to show the convergence of $\left\langle\xi, j_{t}\left(X_{\alpha}\right) \xi\right\rangle$ to $\left\langle\xi, j_{t}(X) \xi\right\rangle$ for arbitrary $\xi$ in $\mathcal{H}$. As $j_{t}\left(X_{\alpha}\right)=F(t) j_{t}\left(X_{\alpha}\right) F(t)$, we can assume $\xi \in \mathcal{H}_{t]}$. Further as the set $\left\{j(\underline{r}, \underline{Y}) u:(\underline{r}, \underline{Y}, u) \in \mathcal{D}_{t]}\right\}$ is total in $\mathcal{H}_{t]}$, it is enough to show the convergence of $\left\langle j(\underline{r}, \underline{Y}) u, j_{t}\left(X_{\alpha}\right) j(\underline{s}, \underline{Z} v)\right\rangle$ for arbitrary $(\underline{r}, \underline{Y}, u),(\underline{s}, \underline{Z}, v) \in$ $\mathcal{D}_{t]}$. In view of (2.7), we can assume $\underline{r}=\underline{s}$. Now observe that,

$$
\left\langle j(\underline{s}, \underline{Y}) u, j_{t}\left(X_{\alpha}\right) j(\underline{s}, \underline{Z}) v\right\rangle=\left\langle u, T_{s_{n}}\left(Y_{n}^{*} \cdots T_{s_{1}-s_{2}}\left(Y_{1}^{*} T_{t-s_{1}}\left(X_{\alpha}\right) Z_{1}\right) \cdots Z_{n}\right) v\right\rangle .
$$

Taking a cue from [Da1] we note that for $A, B, C \in \mathcal{A}_{0}$,

$$
A^{*} C B=\frac{1}{4} \sum_{r=0}^{3}(-i)^{r}\left(A+i^{r} B\right)^{*} C\left(A+i^{r} B\right) .
$$

On applying this formula repeatedly, we have

$$
\begin{gathered}
T_{s_{n}}\left(Y_{n}^{*} \cdots T_{s_{1}-s_{2}}\left(Y_{1}^{*} T_{t-s_{1}}\left(X_{\alpha}\right) Z_{1}\right) \cdots Z_{n}\right) \\
=\frac{1}{4^{n}} \sum_{r_{1}, r_{2}, \ldots r_{n}=0}^{3}(-i)^{\left(\sum_{k=0}^{n} r_{k}\right)} T_{s_{n}}\left(W_{n r_{n}}^{*}\left(\cdots W_{1 r_{1}}^{*} T_{t-s_{1}}\left(X_{\alpha}\right) W_{1 r_{1}} \cdots\right) W_{n r_{n}}\right)
\end{gathered}
$$

where $W_{k r_{k}}=Y_{k}+(i)^{r_{k}} Z_{k}, 1 \leq k \leq n$. Now use normality of $T_{t}$ for all $t$, on the right hand side expression to conclude the convergence of (2.8).

\section{IN DISCRETE TIME}

Here we work with the discrete semigroup formed by integer powers of a single completely positive map on the algebra of all bounded operators on a Hilbert space. We build the dilation and show how it factorizes. Though the constructions here are quite elementary, they are quite instructive and show us as to why one should expect product spaces in continuous time.

Let $\mathcal{H}_{0}$ be a complex separable Hilbert space with the inner-product $\langle.,$.$\rangle anti-$ linear in the first variable. Let $\mathcal{A}_{0}$ be the algebra $\mathcal{B}\left(\mathcal{H}_{0}\right)$. Suppose that $T: \mathcal{A}_{0} \rightarrow \mathcal{A}_{0}$ is a unital, normal completely positive map.

For $u, v$ in $\mathcal{H}_{0}$, let $|u\rangle\langle v|$ be the rank one operator on $\mathcal{H}_{0}$ defined by

$$
|u\rangle\langle v| w=\langle v, w\rangle u, \quad \text { for all } \quad w \in \mathcal{H}_{0} .
$$

Note that $|\rangle.\langle$.$| is linear in first variable and anti-linear in the second. For any$ bounded operator $X$ on $\mathcal{H}_{0}$,

$$
|u\rangle\langle v|=| X u\rangle\langle v|, \quad \text { and } \quad|u\rangle\langle v|X=| u\rangle\left\langle X^{*} v\right| .
$$

Also note that if $u_{n} \rightarrow u$, and $v_{n} \rightarrow v$ in $\mathcal{H}_{0}$ then $\left|u_{n}\right\rangle\left\langle v_{n}\right|$ converges in norm to $|u\rangle\langle v|$.

Now we define two positive definite kernels $([\mathrm{Pa}])$ and via the GNS theory obtain two Hilbert spaces, which will act as building blocks for the dilation space. Let $\mathcal{K}$, $\mathcal{L}$ be the Cartesian products $\mathcal{K}=\mathcal{H}_{0} \times \mathcal{H}_{0} \times \mathcal{H}_{0}$ and $\mathcal{L}=\mathcal{H}_{0} \times \mathcal{H}_{0}$. Define $K: \mathcal{K} \times \mathcal{K} \rightarrow \mathbb{C}$ and $L: \mathcal{L} \times \mathcal{L} \rightarrow \mathbb{C}$ by

$K\left(\left(a_{1}, b_{1}, c_{1}\right),\left(a_{2}, b_{2}, c_{2}\right)\right)=\left\langle c_{1},\left(T\left(\left|a_{1}\right\rangle\left\langle\left. b_{1}\right|^{*} \mid a_{2}\right\rangle\left\langle b_{2}\right|\right)-T\left(\left|a_{1}\right\rangle\left\langle b_{1}\right|\right)^{*} T\left(\left|a_{2}\right\rangle\left\langle b_{2}\right|\right)\right) c_{2}\right\rangle$,

and 


$$
L\left(\left(a_{1}, b_{1}\right),\left(a_{2}, b_{2}\right)\right)=\left\langle b_{1}, T\left(\left|a_{1}\right\rangle\left\langle a_{2}\right|\right) b_{2}\right\rangle
$$

for $a_{1}, b_{1}, c_{1}, a_{2}, b_{2}, c_{2}$ in $\mathcal{H}_{0}$.

Proposition 3.1. Let $K$ and $L$ be as above. Then $K, L$ are positive definite kernels on $\mathcal{K}, \mathcal{L}$ respectively.

Proof. Consider $z_{i} \in \mathbb{C},\left(a_{i}, b_{i}, c_{i}\right) \in \mathcal{K}$ for $1 \leq i \leq n$, for some $n$. On taking $X_{i}=\left|a_{i}\right\rangle\left\langle b_{i}\right|$, we have

$$
\sum_{i, j=1}^{n} \bar{z}_{i} z_{j} K\left(\left(a_{i}, b_{i}, c_{i}\right),\left(a_{j}, b_{j}, c_{j}\right)\right)=\sum_{i, j=1}^{n} \bar{z}_{i} z_{j}\left\langle c_{i},\left(T\left(X_{i}^{*} X_{j}\right)-T\left(X_{i}\right)^{*} T\left(X_{j}\right)\right) c_{j}\right\rangle
$$

which can be rewritten as

$$
\sum_{i, j=1}^{2 n} \bar{w}_{i} w_{j}\left\langle d_{i}, T\left(Y_{i}^{*} Y_{j}\right) d_{j}\right\rangle
$$

where $w_{i}=w_{i+n}=z_{i}, d_{i}=c_{i}, d_{i+n}=-T\left(X_{i}\right) c_{i}, Y_{i}=X_{i}, Y_{i+n}=I$ for $1 \leq i \leq n$. Now the non-negativity of (3.1) follows from complete positivity of $T$.

For a fixed unit vector $a$ in $\mathcal{H}_{0}$, denote the operator $|a\rangle\left\langle a_{i}\right|$ by $Z_{i}$. Then

$$
\sum \bar{z}_{i} z_{j} L\left(\left(a_{i}, b_{i}\right),\left(a_{j}, b_{j}\right)\right)=\sum \bar{z}_{i} z_{j}\left\langle b_{i}, T\left(\left|a_{i}\right\rangle\left\langle a_{j}\right|\right) b_{j}\right\rangle=\sum \bar{z}_{i} z_{j}\left\langle b_{i}, T\left(Z_{i}^{*} Z_{j}\right) b_{j}\right\rangle .
$$

Once again the complete positivity of $T$ gives the required result.

Proposition 3.2. There exists Hilbert spaces $\mathcal{N}$ and $\mathcal{P}$ with maps $\lambda: \mathcal{K} \rightarrow \mathcal{N}$, and $\delta: \mathcal{L} \rightarrow \mathcal{P}$, satisfying

(i) $\left\langle\lambda\left(a_{1}, b_{1}, c_{1}\right), \lambda\left(a_{2}, b_{2}, c_{2}\right)\right\rangle$

$$
=\left\langle c_{1},\left(T\left(\left|a_{1}\right\rangle\left\langle\left. b_{1}\right|^{*} \mid a_{2}\right\rangle\left\langle b_{2}\right|\right)-T\left(\left|a_{1}\right\rangle\left\langle b_{1}\right|\right)^{*} T\left(\left|a_{2}\right\rangle\left\langle b_{2}\right|\right)\right) c_{2}\right\rangle,
$$

$\left\langle\delta\left(a_{1}, b_{1}\right), \delta\left(a_{2}, b_{2}\right)\right\rangle=\left\langle b_{1}, T\left(\left|a_{1}\right\rangle\left\langle a_{2}\right|\right) b_{2}\right\rangle$.

(ii) The sets $\lambda(\mathcal{K})$ and $\delta(\mathcal{L})$ are total (linear combinations form a dense set) in $\mathcal{N}$ and $\mathcal{P}$ respectively.

(iii) The Hilbert spaces $\mathcal{N}$ and $\mathcal{P}$ are separable.

Proof. Existence of Hilbert spaces satisfying (i) and (ii) follows from GNS theory and positive definiteness of $K$ and $L$. Separability of $\mathcal{N}$ and $\mathcal{P}$ is immediate from separability of $\mathcal{H}_{0}$ and easily derivable estimates,

$$
\|\lambda(a, b, c)\|^{2} \leq 2\|\mid a\|^{2}\|b\|^{2}\|c\|^{2} \quad \text { and } \quad\|\delta(a, b)\|^{2} \leq\|a\|\left\|^{2}\right\| b \|^{2}
$$

for $a, b, c$ in $\mathcal{H}_{0}$.

Dimensions of $\mathcal{N}$ and $\mathcal{P}$ are important numerical invariants for the semigroup $\left\{T^{n}\right\}$. In fact, the dimension of $\mathcal{P}$ can be thought of as the numerical index of the semigroup in discrete time. Clearly, $\mathcal{N}$ is zero if and only if $T$ is an endomorphism. So in a sense, dimension of $\mathcal{N}$ measures the deviation of $T$ from an endomorphism. It might be mentioned that every normal completely positive map on $\mathcal{B}\left(\mathcal{H}_{0}\right)$ can be represented in the form $\sum L_{i} X L_{i}^{*}$ for some bounded operators $L_{i}$, and $\operatorname{dim}(\mathcal{P})$ is just the minimum number of $L_{i}$ 's needed to represent $T$ in this form. 
Now we recast Stinespring's theorem [St] in a form more suitable for us.

Theorem 3.3. There exists a unitary map $V: \mathcal{H}_{0} \oplus \mathcal{N} \rightarrow \mathcal{H}_{0} \otimes \mathcal{P}$ such that

$$
\left.V^{*}(X \otimes I) V\right|_{\mathcal{H}_{0}}=T(X)
$$

for $X \in \mathcal{B}\left(\mathcal{H}_{0}\right)$, and the closed linear span of $\left\{(X \otimes I) V u: u \in \mathcal{H}_{0}, X \in \mathcal{B}\left(\mathcal{H}_{0}\right)\right\}$ is $\mathcal{H}_{0} \otimes \mathcal{P}$.

Proof. Fix an orthonormal basis $\left\{e_{0}, e_{1}, \ldots\right\}$ of $\mathcal{H}_{0}$. We first define $V$ on $\mathcal{H}_{0} \subset$ $\mathcal{H}_{0} \oplus \mathcal{N}$ by setting

$$
V x=\sum_{n} e_{n} \otimes \delta\left(e_{n}, x\right)
$$

for $x \in \mathcal{H}_{0}$. For any finite subset $E$ of $\left\{e_{0}, e_{1}, \ldots\right\}$

$$
\begin{aligned}
\left\langle\sum_{n \in E} e_{n} \otimes \delta\left(e_{n}, x\right), \sum_{m \in E} e_{m} \otimes \delta\left(e_{m}, y\right)\right\rangle & =\sum_{n \in E}\left\langle\delta\left(e_{n}, x\right), \delta\left(e_{n}, y\right)\right\rangle \\
& =\left\langle x, T\left(\sum_{n \in E}\left|e_{n}\right\rangle\left\langle e_{n}\right|\right) y\right\rangle .
\end{aligned}
$$

By normality of $T$ it is clear that $V$ is well-defined (i.e., the series in (3.3) converges) and is an isometry on $\mathcal{H}_{0}$. Also note that for $X \in \mathcal{B}\left(\mathcal{H}_{0}\right)$,

$\langle V x,(X \otimes I) V y\rangle=\left\langle\sum_{n} e_{n} \otimes \delta\left(e_{n}, x\right), \sum_{m} X e_{m} \otimes \delta\left(e_{m}, y\right)\right\rangle=\lim _{k \rightarrow \infty}\left\langle x, T\left(P_{k} X P_{k}\right) y\right\rangle$, where $P_{k}$ is the projection onto the span of $\left\{e_{0}, e_{1}, \ldots, e_{k}\right\}$. Once again by normality of $T$

$$
\langle V x,(X \otimes I) V y\rangle=\langle x, T(X) y\rangle .
$$

Now extend $V$ to $\mathcal{H}_{0} \oplus \mathcal{N}$ by taking

$$
V\left(x \oplus \lambda\left(a_{1}, b_{1}, c_{1}\right)\right)=V x+\left(X_{1} \otimes I\right) V c_{1}-V T\left(X_{1}\right) c_{1}
$$

where $X_{1}=\left|a_{1}\right\rangle\left\langle b_{1}\right|, a_{1}, b_{1}, c_{1} \in \mathcal{H}_{0}$. An elementary computation using (3.4) yields

$$
\begin{aligned}
\langle V x & \left.+\left(X_{1} \otimes I\right) V c_{1}-V T\left(X_{1}\right) c_{1}, V y+\left(X_{2} \otimes I\right) V c_{2}-V T\left(X_{2}\right) c_{2}\right\rangle \\
& =\langle x, y\rangle+\left\langle c_{1},\left(T\left(X_{1}^{*} X_{2}\right)-T\left(X_{1}^{*}\right) T\left(X_{2}\right)\right) c_{2}\right\rangle .
\end{aligned}
$$

Recalling the definition of $\lambda$ it is clear that $V$ extends as an isometric operator to whole of $\mathcal{H}_{0} \oplus \mathcal{N}$. Note that

$$
\begin{aligned}
V\left(-T\left(X_{1}\right) c_{1} \oplus \lambda\left(a_{1}, b_{1}, c_{1}\right)\right) & =\left(X_{1} \otimes I\right) V c_{1} \\
& =\sum_{n}\left|a_{1}\right\rangle\left\langle b_{1}\right| e_{n} \otimes \delta\left(e_{n}, c_{1}\right) \\
& =a_{1} \otimes \sum_{n}\left\langle b_{1}, e_{n}\right\rangle \delta\left(e_{n}, c_{1}\right) \\
& =a_{1} \otimes \delta\left(b_{1}, c_{1}\right) .
\end{aligned}
$$

Hence $V$ maps $\mathcal{H}_{0} \oplus \mathcal{N}$ onto $\mathcal{H}_{0} \otimes \mathcal{P}$ and $\left\{\left(X_{1} \otimes I\right) V c_{1}: X_{1}=\left|a_{1}\right\rangle\left\langle b_{1}\right|, a_{1}, b_{1}, c_{1} \in \mathcal{H}_{0}\right\}$ is total in $\mathcal{H}_{0} \otimes \mathcal{P}$.

Remark 3.4. If $\mathcal{H}_{0}$ is finite dimensional then so are $\mathcal{N}$ and $\mathcal{P}$. Then Theorem 3.3 implies $\operatorname{dim}(\mathcal{N})=\operatorname{dim}\left(\mathcal{H}_{0}\right)(\operatorname{dim} \mathcal{P}-1)$. No such formula holds in the infinite dimensional case. 
We shall realize the minimal dilation of the semigroup $\left\{T^{n}, n \geq 0\right\}$ on the Hilbert space

$$
\mathcal{H}=\mathcal{H}_{0} \oplus \mathcal{N} \oplus(\mathcal{N} \otimes \mathcal{P}) \oplus\left(\mathcal{N} \oplus \mathcal{P}^{\otimes^{2}}\right) \oplus \cdots
$$

Let $F(n)$ denote the orthogonal projection of $\mathcal{H}$ onto $\mathcal{H}_{n]}$ where $\mathcal{H}_{0]}=\mathcal{H}_{0}, \mathcal{H}_{1]}=$ $\mathcal{H}_{0} \oplus \mathcal{N}$, and

$$
\mathcal{H}_{n]}=\mathcal{H}_{0} \oplus \mathcal{N} \oplus(\mathcal{N} \otimes \mathcal{P}) \oplus \cdots \oplus\left(\mathcal{N} \otimes \mathcal{P}^{\otimes^{(n-1)}}\right)=\mathcal{H}_{n-1]} \oplus\left(\mathcal{N} \otimes \mathcal{P}^{(n-1)}\right)
$$

for $n \geq 2$, thought of as subspaces of $\mathcal{H}$ in the natural way. Observe that on taking

$$
\mathcal{N}_{n}=\mathcal{N} \oplus(\mathcal{N} \otimes \mathcal{P}) \oplus \cdots \oplus\left(\mathcal{N} \otimes \mathcal{P}^{\otimes^{(n-1)}}\right)
$$

we have $\mathcal{H}_{n]}=\mathcal{H}_{0} \oplus \mathcal{N}_{n}$, and $\mathcal{N}_{m+n} \cong \mathcal{N}_{m} \oplus\left(\mathcal{N}_{n} \otimes \mathcal{P}^{m}\right)$.

Now we obtain a family of unitary operators $V_{n}: \mathcal{H}_{n]} \rightarrow \mathcal{H}_{0} \oplus \mathcal{P} \otimes^{n}$ by induction. Take $V_{1}: \mathcal{H}_{0} \oplus \mathcal{N} \rightarrow \mathcal{H}_{0} \otimes \mathcal{P}$ to be the operator $V$ of Theorem 3.3. Let $I_{\mathcal{N}}, I_{n}$ be identity operators on $\mathcal{N}$ and $\mathcal{P}^{\otimes^{n}}$ respectively. Now if $V_{n-1}$ maps $\mathcal{H}_{n+1]}$ to $\mathcal{H}_{0} \otimes \mathcal{P}^{\otimes^{(n-1)}}$, then $\left(V_{n-1} \oplus\left(I_{\mathcal{N}} \otimes I_{n-1}\right)\right)$ maps $\mathcal{H}_{n-1]} \oplus\left(\mathcal{N} \otimes \mathcal{P}^{\otimes^{(n-1)}}\right)$ to $\left(\mathcal{H}_{0} \otimes\right.$ $\left.\mathcal{P}^{\otimes^{(n-1)}}\right) \oplus\left(\mathcal{N} \otimes \mathcal{P}^{\otimes^{(n-1)}}\right)$. But

$$
\left(\mathcal{H}_{0} \otimes \mathcal{P}^{\otimes^{(n-1)}}\right) \oplus\left(\mathcal{N} \otimes \mathcal{P}^{\otimes^{(n-1)}}\right) \cong\left(\mathcal{H}_{0} \oplus \mathcal{N}\right) \otimes \mathcal{P}^{\otimes^{(n-1)}}
$$

in a natural way. Using this identification, define $V_{n}: \mathcal{H}_{n]}=\mathcal{H}_{n-1]} \oplus(\mathcal{N} \otimes$ $\left.\mathcal{P}^{\otimes^{(n-1)}}\right) \rightarrow \mathcal{H}_{0} \otimes \mathcal{P}^{\otimes^{n}}$ by

$$
V_{n}=\left(V \otimes I_{n-1}\right)\left(V_{n-1} \oplus\left(I_{\mathcal{N}} \otimes I_{n-1}\right)\right)
$$

for $n \geq 2$. Clearly $V_{n}$ is a unitary operator as it is a composition of two unitary operators.

Theorem 3.5. Let $\mathcal{H}, F=\{F(n)\}$ be as above. Define representations $j=\left\{j_{n}\right\}$ of $\mathcal{B}\left(\mathcal{H}_{0}\right)$ in $\mathcal{B}(\mathcal{H})$ by $j_{0}(X)=X F(0)$,

$$
j_{n}(X)=V_{n}^{*}\left(X \otimes I_{n}\right) V_{n} F(n) \text { for } n \geq 1,
$$

and $X \in \mathcal{B}\left(\mathcal{H}_{0}\right)$. Then $(\mathcal{H}, F, j)$ is a minimal Markov dilation of semigroup $\left\{T^{n}, n \geq\right.$ $0\}$.

Proof. First we verify the Markov property. We have

$$
\begin{aligned}
F(n-1) & j_{n}(X) F(n-1) \\
= & F(n-1)\left[V_{n-1}^{*} \oplus\left(I_{\mathcal{N}} \otimes I_{n-1}\right)\right]\left(V^{*} \oplus I_{n-1}\right)\left(X \otimes I_{n}\right) \\
& \times\left(V \otimes I_{n-1}\right)\left[V_{n-1}^{*} \oplus\left(I_{\mathcal{N}} \otimes I_{n-1}\right)\right] F(n-1) \\
= & F(n-1) V_{n-1}^{*}\left(V^{*} \oplus I_{n-1}\right)\left(X \otimes I_{n}\right)\left(V \otimes I_{n-1}\right) V_{n-1} F(n-1) .
\end{aligned}
$$

Note that the range of $V_{n-1} F(n-1)$ is $\mathcal{H}_{0} \otimes \mathcal{P}^{\otimes^{(n-1)}} \subset\left(\mathcal{H}_{0} \oplus \mathcal{N}\right) \otimes \mathcal{P}^{\otimes^{(n-1)}}$, and from Theorem 3.3

$$
\left.\left(V^{*} \oplus I_{n-1}\right)\left(X \otimes I_{n}\right)\left(V \otimes I_{n-1}\right)\right|_{\mathcal{H}_{0} \otimes \mathcal{P} \otimes}{ }^{(n-1)}=T(X) \otimes I_{n-1} .
$$

Hence $F(n-1) j_{n}(X) F(n-1)=F(n-1) V_{n-1}^{*}\left(T(X) \otimes I_{n-1}\right) V_{n-1} F(n-1)=$ $j_{n-1}(T(X))$. Now to prove minimality, it is enough to show that $\left\{j_{n}(X) \xi: \xi \in\right.$ $\mathcal{H}_{n-1]}=$ range of $\left.F(n-1), X \in \mathcal{B}\left(\mathcal{H}_{0}\right)\right\}$ spans $\mathcal{H}_{n]}$ for every $n \geq 1$. We have

$$
j_{n}(X) F(n-1)=V_{n}^{*}\left(X \otimes I_{n}\right)\left(V \otimes I_{n-1}\right) V_{n-1} F(n-1) .
$$


As $V_{n-1}, V_{n}^{*}$ are unitary operators and the closed linear span of $\{(X \otimes I) V u: u \in$ $\left.\mathcal{H}_{0}, X \in \mathcal{B}\left(\mathcal{H}_{0}\right)\right\}$ is $\mathcal{H}_{0} \otimes \mathcal{P}$,

$$
\begin{aligned}
\overline{\operatorname{span}} & \left\{V_{n}^{*}\left(X \otimes I_{n}\right)\left(V \otimes I_{n-1}\right) V_{n-1} \xi: \xi \in \mathcal{H}_{n-1]}, X \in \mathcal{B}\left(\mathcal{H}_{0}\right)\right\} \\
& =\overline{\operatorname{span}}\left\{V_{n}^{*}\left(X \otimes I_{n}\right)\left(V \otimes I_{n-1}\right) \eta: \eta \in \mathcal{H}_{0} \otimes \mathcal{P}^{\otimes^{(n-1)}}, X \in \mathcal{B}\left(\mathcal{H}_{0}\right)\right\} \\
& =\overline{\operatorname{span}}\left\{V_{n}^{*} \gamma: \gamma \in \mathcal{H}_{0} \otimes \mathcal{P}^{\otimes^{n}}\right\} \\
& =\mathcal{H}_{n]} . \quad \square
\end{aligned}
$$

Now notice that $V_{n}^{*}$ maps $\mathcal{H}_{0} \otimes \mathcal{P}^{\otimes^{n}}$ to $\mathcal{H}_{n]}$. By making the obvious identification of $\mathcal{H}_{0}^{\perp} \otimes \mathcal{P}^{\otimes^{n}}$ with $\mathcal{H}_{n]}^{\perp}$, extend $V_{n}^{*}$ to a unitary operator from $\mathcal{H} \otimes \mathcal{P}^{\otimes^{n}}$. It is easily verified that $\theta=\left\{\theta_{n}\right\}$ defined by

$$
\theta_{n}(Z)=W_{n}\left(Z \otimes I_{n}\right) W_{n}^{*}, \quad Z \in \mathcal{B}(\mathcal{H}),
$$

is a semigroup of endomorphisms of $\mathcal{B}(\mathcal{H})$, satisfying

$$
\theta_{n}(X F(0))=j_{n}(X),
$$

for $X \in \mathcal{B}\left(\mathcal{H}_{0}\right)$. Observe that $\theta$ has the property $\theta_{m}\left(j_{n}(X)\right)=j_{m+n}(X)$.

\section{BASIC CONSTRUCTIONS}

In this section we are considering continuous time (i.e., $\mathbb{T}_{+}=\mathbb{R}_{+}$) conservative quantum dynamical semigroups on a type I factor $\mathcal{A}_{0}=\mathcal{B}\left(\mathcal{H}_{0}\right)$, for a complex separable Hilbert space $\mathcal{H}_{0}$. Fix one such semigroup $T$. Throughout we assume that the maps $T_{t}, t \geq 0$, are normal. Let $(\mathcal{H}, F, j)$ be the minimal dilation of the semigroup $T$ provided by Theorem 2.3 .

We shall construct two families of subspaces of $\mathcal{H},\left\{\mathcal{N}_{t}, t \geq 0\right\}$ and $\left\{\mathcal{P}_{t}, t \geq 0\right\}$, satisfying

(i) $\mathcal{P}_{s+t} \cong \mathcal{P}_{s} \otimes \mathcal{P}_{t}$

(ii) $\mathcal{N}_{s+t} \cong \mathcal{N}_{s} \oplus\left(\mathcal{N}_{t} \otimes \mathcal{P}_{s}\right)$;

(iii) $\mathcal{H}_{t]}=\mathcal{H}_{0} \oplus \mathcal{N}_{t} \cong \mathcal{H}_{0} \otimes \mathcal{P}_{t}$,

in a canonical way. Moreover just as in the discrete time, unitary operators $V(t)$ : $\mathcal{H}_{0} \oplus \mathcal{N}_{t} \rightarrow \mathcal{H}_{0} \otimes \mathcal{P}_{t}$, implementing the isomorphism in (iii), will factorize the flow. That is, we see that

$$
j_{t}(X)=V(t)^{*}(X \otimes I) V(t) F(t), \text { for } X \in \mathcal{A}_{0}, t \geq 0
$$

holds where $I_{t}$ is the identity operator on $\mathcal{P}_{t}$. Of course, in the final analysis $\left\{\mathcal{P}_{t}\right\}$ will give us a product system in the sense of Arveson.

Now as the algebra under consideration is $\mathcal{B}\left(\mathcal{H}_{0}\right)$, and the maps $T_{t}$ are assumed to be normal, action of the flow on rank one operators has full information about the flow. In more precise terms we have the following proposition.

Proposition 4.1. The set $\left\{j(\underline{r}, \underline{Y}) u ;(\underline{r}, \underline{Y}, u) \in \mathcal{D}_{t]}\right.$, with $Y_{i}$ 's of the form $\left|x_{i}\right\rangle\left\langle y_{i}\right|$ for some $\left.x_{i}, y_{i} \in \mathcal{H}_{0}\right\}$ is total in $\mathcal{H}_{t]}$.

Proof. Any finite rank operator is a finite linear combination of operators of the form $\left|x_{i}\right\rangle\left\langle y_{i}\right|$. Now normality of $j_{t}$ and a simple induction argument show that we can approximate all vectors of the form $j(\underline{r}, \underline{Y}) u$ with $Y_{i} \geq 0$. Clearly that is good enough. 
Here is an elementary but key observation. For arbitrary $x_{1}, x_{2}, y_{1}, y_{2} \in \mathcal{H}_{0}$ and $\xi, \eta \in \mathcal{H}$

$\left\langle j_{t}\left(\left|x_{1}\right\rangle\left\langle x_{2}\right|\right) \xi, j_{t}\left(\left|y_{1}\right\rangle\left\langle y_{2}\right|\right) \eta\right\rangle=\left\langle\xi, j_{t}\left(\left|x_{2}\right\rangle\left\langle x_{1}|| y_{1}\right\rangle\left\langle y_{2}\right|\right) \eta\right\rangle=\left\langle x_{1}, y_{1}\right\rangle\left\langle\xi, j_{t}\left(\left|x_{2}\right\rangle\left\langle y_{2}\right|\right) \eta\right\rangle$.

As a consequence for an arbitrary unit vector $a$ in $\mathcal{H}_{0}$,

$$
\left\langle j_{t}\left(\left|x_{1}\right\rangle\left\langle x_{2}\right|\right) \xi, j_{t}\left(\left|y_{1}\right\rangle\left\langle y_{2}\right|\right) \eta\right\rangle=\left\langle x_{1}, y_{1}\right\rangle\left\langle j_{t}\left(|a\rangle\left\langle x_{2}\right|\right) \xi, j_{t}\left(|a\rangle\left\langle y_{2}\right|\right) \eta\right\rangle \text {. }
$$

Proposition 4.2. For a unit vector $a \in \mathcal{H}_{0}$, let $\mathcal{P}_{t}(a)$ denote the range of $j_{t}(|a\rangle\langle a|)$. Then

(i) $\mathcal{P}_{t}(a)=$ Range $j_{t}(|a\rangle\langle x|)$ for any $x \neq 0$ in $\mathcal{H}_{0}$.

(ii) If $b$ is another unit vector in $\mathcal{H}_{0}$ then $\mathcal{P}_{t}(a) \cong \mathcal{P}_{t}(b)$.

Proof. Observe

$$
\begin{gathered}
j_{t}(|a\rangle\langle a|) j_{t}(|a\rangle\langle x|)=j_{t}(|a\rangle\langle x|), \\
j_{t}(|a\rangle\langle x|) j_{t}(|x\rangle\langle a|)=\langle x, x\rangle j_{t}(|a\rangle\langle a|) .
\end{gathered}
$$

So the range of $j_{t}(|a\rangle\langle x|)$ is contained in, and contains $\mathcal{P}_{t}(a)$. Now define $Z$ : $\mathcal{P}_{t}(a) \rightarrow \mathcal{P}_{t}(b)$ by putting

$$
Z j_{t}(|a\rangle\langle a|) \xi=j_{t}(|b\rangle\langle a|) \xi \text { for } \xi \in \mathcal{H}
$$

Then (4.2) shows that $Z$ is an isometry and from (i) $Z$ is onto. Hence $Z$ defines a unitary isomorphism between $\mathcal{P}_{t}(a)$ and $\mathcal{P}_{t}(b)$.

Now choose and fix a unit vector $a$ in $\mathcal{H}_{0}$. This vector $a$ is fixed once and for all. Define $\mathcal{P}_{t}$ by

$$
\mathcal{P}_{t}=\mathcal{P}_{t}(a)=\text { Range of } j_{t}(|a\rangle\langle a|) .
$$

Note that $\mathcal{P}_{t}$ is a Hilbert space, that is, up to unitary isomorphisms does not depend upon the choice of $a$. Now we obtain a family of unitary operators $W_{s, t}: \mathcal{H}_{s]} \otimes \mathcal{P}_{t} \rightarrow$ $\mathcal{H}_{s+t]}$ by setting

$$
W_{s, t}\left(j(\underline{r}, \underline{Y}) u \otimes j_{t}(|a\rangle\langle x|) \xi\right)=j(\underline{r}+t, \underline{Y}) j_{t}(|u\rangle\langle x|) \xi
$$

for $(\underline{r}, \underline{Y}, u) \in \mathcal{D}_{s]}, x \in \mathcal{H}_{0}, \xi \in \mathcal{H}_{t]}$. Here by $\underline{r}+t$ we mean $\left(r_{1}+t, r_{2}+t, \ldots, r_{n}+t\right)$.

Theorem 4.3. For $s, t \geq 0, W_{s, t}$ defined as above extends to a unitary operator from $\mathcal{H}_{s]} \otimes \mathcal{P}_{t}$ onto $\mathcal{H}_{s+t]}$.

Proof. Consider $(\underline{r}, \underline{Y}, u),(\underline{r}, \underline{Z}, v) \in \mathcal{D}_{s]}, x, y \in \mathcal{H}_{0]}$, and $\xi, \eta \in \mathcal{H}_{t]}$. From (2.2) and (2.3) we have

$$
\begin{aligned}
\langle j(\underline{r}+ & \left.t, \underline{Y}) j_{t}(|u\rangle\langle x|) \xi, j(\underline{r}+t, \underline{Z}) j_{t}(|v\rangle\langle y|) \eta\right\rangle \\
= & \left\langle j_{t}(|u\rangle\langle x|) \xi, j\left(\left(r_{n}, r_{n-1}, \ldots, r_{1}, r_{1}, \ldots, r_{n}\right)+t\right.\right. \\
& \left.\left.\left(Y_{n}^{*}, \ldots, Y_{1}^{*}, Z_{1}, \ldots, Z_{n}\right)\right) j_{t}(|v\rangle\langle y|) \eta\right\rangle \\
& =\left\langle j_{t}(|u\rangle\langle x|) \xi, j_{t}\left(\epsilon\left(r_{n}, \ldots, r_{1}, r_{1}, \ldots r_{n}\right),\left(Y_{n}^{*}, \ldots, Y_{1}, Z_{1}, \ldots, Z_{n}\right)\right) j_{t}(|v\rangle\langle y|) \eta\right\rangle \\
= & \left\langle\xi, j_{t}\left(|x\rangle\left\langle u\left|\epsilon\left(\left(r_{n}, \ldots, r_{1}, r_{1}, \ldots r_{n}\right),\left(Y_{n}^{*}, \ldots, Y_{1}, Z_{1}, \ldots, Z_{n}\right)\right)\right| v\right\rangle\langle y|\right) \eta\right\rangle \\
= & \left.\left\langle u, \epsilon\left(r_{n}, \ldots, r_{1}, r_{1}, \ldots r_{n}\right),\left(Y_{n}^{*}, \ldots, Y_{1}, Z_{1}, \ldots, Z_{n}\right)\right) v\right\rangle .\left\langle\xi, j_{t}(|x\rangle\langle y|) \eta\right\rangle \\
= & \langle j(\underline{r}, \underline{Y}) u, j(\underline{r}, \underline{Z}) v\rangle .\left\langle j_{t}(|a\rangle\langle x|) \xi, j_{t}(|a\rangle\langle y|) \eta\right\rangle .
\end{aligned}
$$

Thus $W_{s, t}$ is isometric. Proposition 4.1 implies that domain and range of $W_{s, t}$ are total in $\mathcal{H}_{s]} \otimes \mathcal{P}_{t}, \mathcal{H}_{s+t]}$ respectively. 
As $\mathcal{P}_{s}$ is a subspace of $\mathcal{H}_{s]}$ we can restrict $W_{s, t}$ to $\mathcal{P}_{s} \otimes \mathcal{P}_{t}$, to have $U_{s, t}: \mathcal{P}_{s} \otimes \mathcal{P}_{t} \rightarrow$ $\mathcal{H}_{s+t]}$, satisfying

$$
U_{s, t}\left(j_{s}(|a\rangle\langle a|) j(\underline{r}, \underline{Y}) u \otimes j_{t}(|a\rangle\langle x|) \xi\right)=j_{s+t}(|a\rangle\langle a|) j(\underline{r}+t, \underline{Y}) j_{t}(|u\rangle\langle x|) \xi
$$

for $(\underline{r}, \underline{Y}, u) \in \mathcal{D}_{s]}, x \in \mathcal{H}_{0}$, and $\xi \in \mathcal{H}_{t]}$. Clearly the range of $U_{s, t}$ is $\mathcal{P}_{s+t}$. So we have unitary operators $U_{s, t}: \mathcal{P}_{s} \otimes \mathcal{P}_{t} \rightarrow \mathcal{P}_{s+t}$. Moreover for $s_{1}, s_{2}, s_{3} \geq 0$, as maps from $\mathcal{H}_{\left.s_{1}\right]} \otimes \mathcal{P}_{s_{2}} \otimes \mathcal{P}_{s_{3}}$ to $\mathcal{H}_{s_{1}+s_{2}+s_{3}}$,

$$
W_{s_{1}, s_{2}+s_{3}}\left(I_{\left.s_{1}\right]} \otimes U_{s_{2}, s_{3}}\right)=W_{s_{1}+s_{2}, s_{3}}\left(W_{s_{1}, s_{2}} \otimes I_{s_{3}}\right)
$$

as is clear from (4.4) and (4.5). (Here for any $s, I_{s]}, I_{s}$ denote identity operator on $\mathcal{H}_{s]}, \mathcal{P}_{s}$, respectively.) On restricting this equality to $\mathcal{P}_{s_{1}} \otimes \mathcal{P}_{s_{2}} \otimes \mathcal{P}_{s_{3}}$, we have the associativity of the tensor product structure on $\left\{\mathcal{P}_{s}\right\}$. That is, as maps from $\mathcal{P}_{s_{1}} \otimes \mathcal{P}_{s_{2}} \otimes \mathcal{P}_{s_{3}}$ to $\mathcal{P}_{s_{1}+s_{2}+s_{3}}$

$$
U_{s_{1}, s_{2}+s_{3}}\left(I_{s_{1}} \otimes U_{s_{2}, s_{3}}\right)=U_{s_{1}+s_{2}, s_{3}}\left(U_{s_{1}, s_{2}} \otimes I_{s_{3}}\right)
$$

for $s_{1}, s_{2}, s_{3} \geq 0$.

Remark 4.4. The definition of $W_{s, t}$ (see (4.4)) can be extended to the case $s=\infty$ by considering $(\underline{r}, \underline{Y}, u)$ in the whole of $\mathcal{D}$. This yields unitary operators $W_{\infty, t}$ : $\mathcal{H} \otimes \mathcal{P}_{t} \rightarrow \mathcal{H},\left(\right.$ recall $\left.\mathcal{H}_{\infty}=\mathcal{H}\right)$ satisfying

$$
W_{\infty, t}(\xi \otimes \eta)=W_{s, t}(\xi \otimes \eta)
$$

for $\xi \in \mathcal{H}_{s]}, \eta \in \mathcal{P}_{t}$. In other words, the operator $W_{s, t}$ is the restriction of $W_{\infty, t}$ to $\mathcal{H}_{s]} \otimes \mathcal{P}_{t}$. Now (4.6) takes the form

$$
W_{\infty, s_{2}+s_{3}}\left(I_{\infty]} \otimes U_{s_{2}, s_{3}}\right)=W_{\infty, s_{3}}\left(W_{\infty, s_{2}} \otimes I_{s_{3}}\right)
$$

as maps from $\mathcal{H} \otimes \mathcal{P}_{s_{2}} \otimes \mathcal{P}_{s_{3}}$ to $\mathcal{H}$, for $s_{2}, s_{3} \geq 0$.

Theorem 4.5. For $t \geq 0$, let $V(t): \mathcal{H}_{t]} \rightarrow \mathcal{H}_{0} \otimes \mathcal{P}_{t}$ be the unitary map $W_{0, t}^{*}$. Then for all $X$ in $\mathcal{B}\left(\mathcal{H}_{0}\right)$

$$
j_{t}(X)=V(t)^{*}\left(X \otimes I_{t}\right) V(t) F(t)
$$

Define $\theta_{t}: \mathcal{B}(\mathcal{H}) \rightarrow \mathcal{B}(\mathcal{H})$ by

$$
\theta_{t}(Z)=W_{\infty, t}\left(Z \otimes I_{t}\right) W_{\infty, t}^{*}, \quad Z \in \mathcal{B}(\mathcal{H}) .
$$

Then $\theta$ is the unique semigroup of normal $*$-endomorphisms of $\mathcal{B}(\mathcal{H})$ satisfying

$$
\theta_{t}(X F(0))=j_{t}(X), \quad X \in \mathcal{B}\left(\mathcal{H}_{0}\right) .
$$

Proof. On taking $V(t)=W_{0, t}^{*}$, from (4.4) (with $s=0$ ) we have

$$
V(t) j_{t}(|u\rangle\langle x|) \xi=u \otimes j_{t}(|a\rangle\langle x|) \xi
$$

for $u, x \in \mathcal{H}_{0}, \xi \in \mathcal{H}_{t]}$. Now if $v, y \in \mathcal{H}_{0}, \eta \in \mathcal{H}_{t]}$, use (4.2) to obtain

$$
\begin{aligned}
\left\langle V(t) j_{t}(|u\rangle\langle x|) \xi,(X \otimes I) j_{t}(|v\rangle\langle y|) \eta\right\rangle & =\left\langle u \otimes j_{t}(|a\rangle\langle x|) \xi, X v \otimes j_{t}(|a\rangle\langle y|) \eta\right\rangle \\
& =\langle u, X v\rangle\left\langle j_{t}(|a\rangle\langle x|) \xi, j_{t}(|a\rangle\langle y|) \eta\right\rangle \\
& =\left\langle j_{t}(|u\rangle\langle x|) \xi, j_{t}(|X v\rangle\langle y|) \eta\right\rangle \\
& =\left\langle j_{t}(|u\rangle\langle x|) \xi, j_{t}(X) j_{t}(|v\rangle\langle y|) \eta\right\rangle .
\end{aligned}
$$


This proves the first claim as vectors of the form $j_{t}(|u\rangle\langle x|) \xi$ are total in $\mathcal{H}_{t]}$. To prove the second part, note that $\theta_{t}$ is an endomorphism due to unitarity of $W_{\infty, t}$. Now on using (4.9),

$$
\begin{aligned}
\theta_{t}\left(\theta_{s}(Z)\right) & =W_{\infty, t}\left(W_{\infty, s} \otimes I_{t}\right)\left(Z \otimes I_{s} \otimes I_{t}\right)\left(W_{\infty, s}^{*} \otimes I_{t}\right) W_{\infty, t}^{*} \\
& =W_{\infty, s+t}\left(I_{\infty]} \otimes U_{s, t}\right)\left(Z \otimes I_{s} \otimes I_{t}\right)\left(I_{\infty]} \otimes U_{s, t}^{*}\right) W_{\infty, s+t}^{*} \\
& =\theta_{s+t}(Z)
\end{aligned}
$$

Finally (4.12) is clear as a restriction of $W_{\infty, t}$ to $\mathcal{H}_{0} \otimes \mathcal{P}_{t}$ is $W_{0, t}$, which is same as $V(t)^{*}$. The uniqueness is proved by showing that (4.12) completely determines $\theta$. Clearly (4.12) implies $\theta_{t}\left(j_{s}(X)\right)=j_{s+t}(X)$ for $X \in \mathcal{B}\left(\mathcal{H}_{0}\right)$. Now for $(\underline{r}, \underline{Y}, u)$, $(\underline{s}, \underline{Z}, v) \in \mathcal{D}$,

$$
\begin{aligned}
\theta_{t}(|j(\underline{r}, \underline{Y}) u\rangle\langle j(\underline{s}, \underline{Z}, v)|) & =\theta_{t}\left(j(\underline{r}, \underline{Y})|u\rangle\langle v| j(\underline{s}, \underline{Z})^{*}\right) \\
& =j(\underline{r}+t, \underline{Y}) \theta_{t}(|u\rangle\langle v|) j(\underline{s}+t, \underline{Z})^{*} \\
& =j(\underline{r}+t, \underline{Y}) j_{t}(|u\rangle\langle v|) j(\underline{s}+t, Z)^{*}
\end{aligned}
$$

Now as the collection $\{j(\underline{r}, \underline{Y}) u ;(\underline{r}, \underline{Y}, u) \in \mathcal{D}\}$ is total and $\theta$ is assumed to be normal, the required result follows.

Observe that on identifying $X \in \mathcal{B}\left(\mathcal{H}_{0}\right)$ with $X F(0)$ in $\mathcal{B}(\mathcal{H})$, (4.12) takes the elegant form $j_{t}(X)=\theta_{t}(X)$, so $\theta$ can be thought of as a dilation of $T$, and as an extension of $j$ to a larger algebra. We do not do this identification here to avoid some confusion (e.g. $\theta_{t}(I)$ would be ambiguous). However as $\mathcal{H}_{0}$ is already thought of as a subspace of $\mathcal{H}$, for $u, v \in \mathcal{H}_{0}$,

$$
j_{t}(|u\rangle\langle v|)=\theta_{t}(|u\rangle\langle v|) .
$$

Now it is not very difficult to see from (4.4) that

$$
U_{s, t}\left(\theta_{s}(|a\rangle\langle a|) \gamma \otimes \theta_{t}(|a\rangle\langle a|) \eta\right)=\theta_{s+t}(|a\rangle\langle a|) \theta_{t}(|\gamma\rangle\langle a|) \eta
$$

for $\gamma, \eta \in \mathcal{H}$.

Remark 4.6. Note that $\theta$ is a quantum dynamical semigroup in its own right. However as it is a semigroup of endomorphisms its dilation is itself. Moreover, (4.13), (4.14) show that its associated family of Hilbert spaces $\mathcal{P}_{t}^{(\theta)}$ and unitary maps $U_{s, t}^{(\theta)}$ are the same as $\mathcal{P}_{t}$ and $U_{s, t}$ respectively.

Perhaps it is worthwhile to restate the existence and uniqueness of 'dilation' $\theta$ of $T$ without referring to intermediate $j$.

Theorem 4.7. Let $T$ be a conservative quantum dynamical semigroup of normal maps on $\mathcal{B}\left(\mathcal{H}_{0}\right)$. Then there exists a pair $(\mathcal{H}, \theta)$, where $\mathcal{H}$ is a Hilbert space containing $\mathcal{H}_{0}$ and $\theta$ is a semigroup of normal $*$-endomorphisms of $\mathcal{B}(\mathcal{H})$, such that if $P$ denotes the orthogonal projection of $\mathcal{H}$ onto $\mathcal{H}_{0}$ then

(i) $\theta_{t}(P)$ is a family of projections increasing to identity with $\theta_{0}(P)=P$;

(ii) $P \theta_{t}(X P) P=T_{t}(X) P$ for $X \in \mathcal{B}\left(\mathcal{H}_{0}\right), t \geq 0$.

(iii) The set $\left\{\theta_{r_{1}}\left(Y_{1} P\right) \theta_{r_{2}}\left(Y_{2} P\right) \cdots \theta_{r_{n}}\left(Y_{n} P\right) u:(\underline{r}, \underline{Y}, u) \in \mathcal{D}\right\}$ is total in $\mathcal{B}(\mathcal{H})$.

If $\left(\mathcal{H}^{\prime}, \theta^{\prime}\right)$ is another such pair then there exists a unitary map $U: \mathcal{H} \rightarrow \mathcal{H}^{\prime}$ such that $U u=u$ for $u \in \mathcal{H}_{0}$, and $\theta^{\prime}(Z)=U \theta\left(U^{*} Z U\right) U^{*}$, for $Z \in \mathcal{B}\left(\mathcal{H}^{\prime}\right)$.

Proof. This is clear from Theorem 4.5 and the uniqueness of the minimal dilation $(\mathcal{H}, F, j)$ up to unitary isomorphisms. 
It may be noted that (ii), (iii) of Theorem 4.7 imply that $\left\{\theta_{t}(P)\right\}$ is a family of projections increasing to identity with $\theta_{0}(P)=P$. More interestingly there is a converse statement to this theorem.

Theorem 4.8. Let $\mathcal{H}$ be a Hilbert space and let $\theta$ be a semigroup of $*$-endomorphisms of $\mathcal{H}$. Suppose $P$ is an orthogonal projection of $\mathcal{H}$ to some subspace $\mathcal{H}_{0}$ such that $P \leq \theta_{t}(P)$ for all $t$. Then $T=\left\{T_{t}\right\}$ defined by

$$
T_{t}(X)=\left.P \theta_{t}(X P) P\right|_{\mathcal{H}_{0}}, \quad X \in \mathcal{B}\left(\mathcal{H}_{0}\right),
$$

is a conservative quantum dynamical semigroup on $\mathcal{B}\left(\mathcal{H}_{0}\right)$.

Proof. Clearly $T_{t}(I)=I$, and $T_{t}$ is completely positive for all $t$. Now for $s, t \geq 0$

$T_{s}\left(T_{t}(X)\right)=\left.P \theta_{s}\left(P \theta_{t}(X P) P\right) P\right|_{\mathcal{H}_{0}}=\left.P \theta_{s}(P) \theta_{s+t}(X P) \theta_{s}(P) P\right|_{\mathcal{H}_{0}}=T_{s+t}(X)$.

This means that we have completely classified (unital, normal) quantum dynamical semigroups on type $I_{n}$ factors in terms of isomorphism classes of triples $(\mathcal{H}, \theta, P)$, where $\mathcal{H}$ is a Hilbert space, $\theta$ is a semigroup of normal $*$-endomorphisms of $\mathcal{B}(\mathcal{H})$, and $P$ is a projection of $\mathcal{H}$ to some subspace $\mathcal{H}_{0}$ of dimension $n$, such that (i), (iii) of Theorem 4.7 are satisfied. This is clearly a semigroup version of Stinespring's theorem.

Now let us look at the example we started with in Section 2. Suppose $T$ is implemented by a strongly continuous semigroup $R$ of contractions. (Actually, coisometries as we assume $T$ to be conservative.) Now from [Bh] one knows that the minimal dilation of $T$ can be obtained from Sz. Nagy's minimal dilation of $R$ to a strongly continuous semigroup $U$ of unitaries. (Exactly as in (2.1) with $V$ replaced by $U$. Minimal isometric dilations of semigroups of co-isometries are automatically unitaries.) With this knowledge it is not hard to see that $\theta$ of $T$ is the semigroup of automorphisms

$$
\theta_{t}(Z)=U_{t} Z U_{t}^{*}
$$

for $t \geq 0, Z \in \mathcal{B}(\mathcal{H})$. Observe that in this case $\mathcal{P}_{t}$ is the one dimensional space spanned by $U_{t} a$ ( $a$ being the unit vector with which we define $\mathcal{P}_{t}$ ).

Conversly, suppose for some semigroup $T, \operatorname{dim} \mathcal{P}_{t} \cong 1$. Then (4.11) implies that $\theta$ is a semigroup of automorphisms of $\mathcal{B}(\mathcal{H})$. Clearly, it can be extended to the negative half-line by setting $\theta_{-t}=\theta_{t}^{-1}$, to have a one parameter group of automorphisms. It is well-known [Va] that strong continuity of $\theta$ forces it to be implemented by a strongly-continuous semigroup of unitaries. Now one can easily deduce that the original semigroup $T$ is also implemented (by a strongly continuous semigroup of contractions).

Now we go back to our program of constructing Hilbert spaces $\left\{\mathcal{N}_{t}\right\}$ as described in the beginning of this section. Actually, the construction is almost a triviality now. As $\mathcal{H}_{t]}, \mathcal{H}_{0}$ are the ranges of $F(t)$ and $F(0)$ with $F(t) \geq F(0)$. Take

$$
\mathcal{N}_{t}=\text { Range of }(F(t)-F(0)) .
$$

It is immediate that $\mathcal{H}_{t]}=\mathcal{H}_{0} \oplus \mathcal{N}_{t}$.

Theorem 4.9. There exists unitary operators $Z_{s, t}$ mapping $\mathcal{N}_{s} \oplus\left(\mathcal{N}_{t} \otimes \mathcal{P}_{s}\right)$ onto $\mathcal{N}_{s+t}$ for $s, t \geq 0$. 
Proof. Observe that $W_{t, s}: \mathcal{H}_{t]} \otimes \mathcal{P}_{s} \rightarrow \mathcal{H}_{t+s]}$ maps the subspace $\mathcal{H}_{0} \otimes \mathcal{P}_{s}$ onto $\mathcal{H}_{s]}$. So clearly it maps $\mathcal{N}_{t} \otimes \mathcal{P}_{s}$ onto the range of $(F(t+s)-F(s))$. Now take $Z_{s, t}$ as $I \oplus W_{t, s}$.

In this theorem we have not bothered to describe the canonicity of associated unitary maps. Observe that $\mathcal{N}_{s_{1}} \oplus\left(\mathcal{N}_{s_{2}} \otimes \mathcal{P}_{s_{1}}\right) \oplus\left(\mathcal{N}_{s_{3}} \oplus \mathcal{P}_{s_{2}+s_{1}}\right) \cong \mathcal{N}_{s_{1}} \oplus\left(\mathcal{N}_{s_{2}} \otimes\right.$ $\left.\mathcal{P}_{s_{1}}\right) \oplus\left(\mathcal{N}_{s_{3}} \otimes \mathcal{P}_{s_{2}} \otimes \mathcal{P}_{s_{1}}\right)$. Now the component Hilbert spaces here can be combined together using unitary operators $Z_{s, t}$ of the theorem to obtain $\mathcal{N}_{s_{1}+s_{2}+s_{3}}$ in two ways. A routine verification shows that the associated diagram commutes.

It maybe noted that the dilation space $\mathcal{H}$ can be decomposed in a simpleminded way as $\mathcal{H}_{0} \oplus \mathcal{N}_{\infty}$ with $\mathcal{N}_{\infty}=\mathcal{H}_{0}^{\perp}$. However in general $\mathcal{H}$ does not admit a natural multiplicative decompositon as $\mathcal{H}_{0} \otimes \mathcal{P}_{\infty}$ through some isomorphism, though as we have seen $\mathcal{H}_{t]} \cong \mathcal{H}_{0} \otimes \mathcal{P}_{t}$ for $0 \leq t<\infty$. There is no natural candidate for $\mathcal{P}_{\infty}$.

As remarked earlier $\mathcal{N}_{t} \cong\{0\}$ for semigroups of endomorphisms. Here is another observation about endomorphisms which could be of some independent interest.

Theorem 4.10. If $T_{t_{0}}$ is an endomorphism of $\mathcal{B}\left(\mathcal{H}_{0}\right)$ for some $t_{0}>0$, then $T$ is a semigroup of endomorphisms.

Proof. Clearly it is enough to show the endomorphism property of $T_{s}$ for $0 \leq s \leq t_{0}$. Fix $s$, and take $s^{\prime}=t_{0}-s, P=F(0), Q=I-P$. With this notation, for $X \in \mathcal{B}\left(\mathcal{H}_{0}\right)$, we have

$$
\begin{aligned}
\left(Q j_{t_{0}}(X) P\right)^{*}\left(Q j_{t_{0}}(X) P\right) & =P j_{t_{0}}\left(X^{*} X\right) P-\left(P j_{t_{0}}\left(X^{*}\right) P\right)\left(P j_{t_{0}}(X) P\right) \\
& =j_{0}\left(T_{s}\left(X^{*} X\right)\right)-j_{0}\left(T_{t_{0}}\left(X^{*}\right)\right) j_{0}\left(T_{t_{0}}(X)\right) \\
& =0 .
\end{aligned}
$$

Hence $Q j_{t_{0}}(X) P=P j_{t_{0}}(X) Q=0$. As $F\left(s^{\prime}\right)$ commutes with $P, Q$, pre and postmultiplication by it gives $Q j_{s^{\prime}}\left(T_{s}(X)\right) P=P j_{s^{\prime}}\left(T_{s}(X)\right) Q=0$. Now for $X, Y \in$ $\mathcal{B}\left(\mathcal{H}_{0}\right)$

$$
\begin{gathered}
j_{0}\left(T_{s^{\prime}}\left(T_{s}(X) T_{s}(Y)\right)\right)=P j_{s^{\prime}}\left(T_{s}(X) T_{s}(Y)\right) P=P j_{s^{\prime}}\left(T_{s}(X)\right)(P+Q) j_{s^{\prime}}\left(T_{s}(Y)\right) P \\
=P j_{s^{\prime}}\left(T_{s}(X)\right) P . P j_{s^{\prime}}\left(T_{s}(Y)\right) P=j_{0}\left(T_{t_{0}}(X) T_{t_{0}}(Y)\right)=j_{0}\left(T_{s^{\prime}}\left(T_{s}(X Y)\right)\right) .
\end{gathered}
$$

That is, $T_{s^{\prime}}\left(T_{s}(X) T_{s}(Y)-T_{s}(X Y)\right)=0$, implying, $T_{t_{0}}\left(T_{s}(X) T_{s}(Y)-T_{s}(X Y)\right)=0$. But then $T_{t_{0}}$ is injective as any representation of the von Neumann algebra $\mathcal{B}\left(\mathcal{H}_{0}\right)$ is the trivial representation with some multiplicity.

It may be noted that the theorem above is applicable to discrete time as well, where it means that if $T^{n}$ is an endomorphism then so is $T$. The continuous time version is not immediate from this as there is no continuity assumption on the semigroup.

\section{REALiZATION OF PRODUCT SYSTEMS}

In this section we study the family of Hilbert spaces $\left\{\mathcal{P}_{t}, t \geq 0\right\}$ in greater detail. To do the analysis of product spaces as in [Ar1, Ar2, Ar3, Ar4, Ar5] one needs associated Hilbert spaces to be separable. This means some regularity assumption on the semigroup. We assume that the semigroup $T$ is such that each $T_{t}, t \geq 0$, is normal and for the associated dilation $\left(\mathcal{H}, F, j_{t}\right)$, the map $t \rightarrow j_{t}(X)$ is strongly continuous for fixed $X \in \mathcal{B}\left(\mathcal{H}_{0}\right)$. Here and elsewhere in this article by strong continuity we mean continuity in strong operator topology. It is convenient to have a definition here. 
Definition 5.1. A quantum dynamical semigroup $\left\{T_{t}\right\}$ is said to be path-continuous if the map $t \rightarrow j_{t}(X)$ is strongly continuous for $X \in \mathcal{B}\left(\mathcal{H}_{0}\right)$.

First verifying for operators of the form $|j(\underline{r}, \underline{Y}) u\rangle\langle j(\underline{v}, \underline{Z}) v|$ as in the proof of the uniqueness of $\theta$, it is not difficult to see that path-continuity implies that the map $t \rightarrow \theta_{t}(Z)$ is also strongly continuous for all $Z \in \mathcal{B}(\mathcal{H})$. We have called this regularity condition path-continuity as the flow $j$ is thought of as a Markov process for the semigroup $T$.

Proposition 5.2. A conservative quantum dynamical semigroup $T$ is path-continuous iff the function

$$
\left\langle u, T_{s_{n}}\left(Y_{n}^{*} \cdots T_{s_{2}}\left(Y_{2} T_{t-s_{2}}\left(T_{s_{1}-t}\left(Y_{1}\right) X T_{s_{1}-t}\left(Z_{1}\right)\right) Z_{2}\right) \cdots Z_{n}\right) v\right\rangle
$$

is continuous in $t$, as $t$ varies in the interval $\left[s_{2}, s_{1}\right]$ for fixed $s_{1}>s_{2}>\cdots>s_{n} \geq 0$ and $X, Y_{1}, \ldots, Y_{n}, Z_{1}, \ldots, Z_{n}$ in $\mathcal{B}\left(\mathcal{H}_{0}\right)$ for $n \geq 2$.

Proof. Assume continuity of functions described above. Firstly, as the $j_{t}$ 's are homomorphisms, once we show weak continuity for all $X$, strong continuity is immediate. Now arguing as in the proof of normality (Proposition 2.4) it is sufficient to show continuity of functions of the form $\left\langle j(\underline{r}, \underline{Y}) u, j_{t}(X) j(\underline{r}, \underline{Z}) v\right\rangle$, for $(\underline{r}, \underline{Y}, u),(\underline{r}, \underline{Z}, v) \in \mathcal{D}$. (Note that we cannot restrict ourselves to $\mathcal{D}_{t}$ as $t$ is varying.) Suppose $t$ varies in a small interval $[a, b] \subset[0, \infty)$. As the semigroup is conservative we can insert arbitrarily large extra time points in $\underline{r}$ and identity operators at corresponding places in $\underline{Y}, \underline{Z}$ and have $r_{1}>r_{2}>\cdots>r_{k} \geq b \geq t \geq$ $a \geq r_{k+1}>\cdots>r_{m} \geq 0$, for some $k$. Now by a repeated application of reduction algorithm we have

$$
\begin{aligned}
j_{t}(X) j\left(\left(r_{1}, \ldots, r_{k+1}\right),\left(Z_{1}, \ldots, Z_{k+1}\right)\right) & =j_{t}(X) j_{r_{k}}(W) j_{r_{k+1}}\left(Z_{k+1}\right) \\
& =j_{t}\left(X T_{r_{k}-t}(W)\right) j_{r_{k+1}}\left(Z_{k+1}\right)
\end{aligned}
$$

for some $W \in \mathcal{B}\left(\mathcal{H}_{0}\right)$. ( $W$ does not depend upon $t$.) In a similar way,

$$
\begin{gathered}
j\left(\left(r_{1}, \ldots, r_{k+1}\right),\left(Y_{1}, \ldots, Y_{k+1}\right)\right)^{*} j_{t}\left(X T_{r_{k}-t}(W)\right) j_{r_{k+1}}\left(Z_{k+1}\right) \\
\quad=j_{r_{k+1}}\left(Y_{k+1}^{*}\right) j_{t}\left(T_{r_{k}-t}(V) X T_{r_{k}-t}(W)\right) j_{r_{k+1}}\left(Z_{k+1}\right) \\
\quad=j_{r_{k+1}}\left(Y_{k+1}^{*} T_{t-r_{k+1}}\left(T_{r_{k}-t}(V) X T_{r_{k}-t}(W)\right) Z_{k+1}\right)
\end{gathered}
$$

for some $V \in \mathcal{B}\left(\mathcal{H}_{0}\right)$. Now it should be clear that $\left\langle j(\underline{r}, \underline{Y}) u, j_{t}(X) j(\underline{r}, \underline{Z}) v\right\rangle=$ $\left\langle u, j(\underline{r}, \underline{Y})^{*} j_{t}(X) j(\underline{r}, \underline{Z}) v\right\rangle$ is of the form (5.1) on suitable renaming of indices.

The converse part should be clear as strong continuity implies weak continuity, and $(5.1)$ is equal to $\left\langle j(\underline{s}, \underline{Y}) u, j_{t}(X) j(\underline{s}, \underline{Z}) v\right\rangle$.

The following proposition lists some important examples of semigroups having this continuity property.

Proposition 5.3. In each of the following cases semigroup $T$ is path-continuous.

(i) $T$ is a semigroup of endomorphisms and $\left\langle u, T_{t}(X) v\right\rangle$ is continuous in $t$ for fixed $u, v \in \mathcal{H}_{0}$ and $X \in \mathcal{B}\left(\mathcal{H}_{0}\right)$.

(ii) $\lim _{t \rightarrow s}\left\|T_{t}(X)-T_{s}(X)\right\|=0$ for every $X$ in $\mathcal{B}\left(\mathcal{H}_{0}\right)$.

(iii) For fixed $X, T_{t}(X)$ converges strongly to $T_{s}(X)$ as $t$ converges to $s$, and for fixed $t, T_{t}\left(X_{r}\right)$ converges strongly to $T_{t}\left(X_{s}\right)$ as $X_{r}$ converges strongly to $X_{r}$ in $\mathcal{B}\left(\mathcal{H}_{0}\right)$.

(iv) $T_{t}(X)=R(t) X R(t)^{*}$ for a strongly continuous semigroup of contractions $R(t)$ on the Hilbert space. 
Proof. In case (i), observe that endomorphism property of $T$ implies,

$$
T_{t-s_{2}}\left(T_{s_{1}-t}\left(Y_{1}\right) X T_{s_{1}-t}\left(Z_{1}\right)\right)=T_{s_{1}-s_{2}}\left(Y_{1}\right) T_{t}(X) T_{s_{1}-s_{2}}\left(Z_{1}\right) .
$$

Now (4.1) reduces to $\left\langle x, T_{t}(X) y\right\rangle$ for some vectors $x, y$ in $\mathcal{H}_{0}$. For second case one has to simply use joint continuity of multiplication of operators in norm. (iii) is clear once we notice that here the map $t \rightarrow T_{t}(Y) X T_{t}(Z)$ is continuous due to contractivity of $T$. And (iv) is as follows from (iii) as $R(t)^{*}$ is also a strongly continuous semigroup (see [Da3]).

Here after we are considering a conservative quantum dynamical semigroup $T$ on $\mathcal{B}\left(\mathcal{H}_{0}\right)$, such that each $T_{t}$ is normal and $T$ is path-continuous. Let $(\mathcal{H}, F, j)$ be the assoicated minimal Markov flow.

Proposition 5.4. The Hilbert space $\mathcal{H}$ is separable.

Proof. Fix a complete orthonormal basis $E$ of $\mathcal{H}_{0}$. Consider the countable set $\Gamma=$ $\left\{\sum_{i=1}^{n} z_{i} j\left(\underline{r_{i}}, \underline{Y_{i}}\right) u_{i}\right\}$, where the $z_{i}$ 's are complex numbers with rational coordinates, $r_{i}$ 's are tuples of positive rational numbers, $Y_{i}$ 's are tuples of operators of the form $\left|x_{i}\right\rangle\left\langle y_{i}\right|$ with $x_{i}, y_{i} \in E$, and also $u_{i} \in E$ for all $i$. Now use strong continuity of the flow to approximate arbitrary time points through rational time points and then use Proposition 4.1 to approximate every vector in $\mathcal{H}_{t]}$. Now recall that due to minimality of the flow, $\bigcup \mathcal{H}_{t]}=\mathcal{H}$.

The technical preparations are over and we move on to explaining the notion of product systems. The paradigm examples of continuous tensor products of Hilbert spaces are obtained through Fock spaces. Let $\mathcal{K}$ be a complex separable Hilbert space. Take

$$
\mathcal{H}_{t}=\Gamma\left(L^{2}([0, t], \mathcal{K})\right)
$$

for $t>0$. That is, $\mathcal{H}_{t}$ is the Boson Fock space over the $L^{2}$ space of $\mathcal{K}$ valued functions on $[0, t]$. Now it is clear that right-shift on $\mathbb{R}_{+}$gives rise to canonical isomorphisms satisfying

$$
\mathcal{H}_{s} \otimes \mathcal{H}_{t} \cong \mathcal{H}_{s+t}
$$

for $s, t>0$. Generalising this, continuous tensor product system of Hilbert spaces has been defined by Arveson [Ar1] as a measurable family $\left\{\mathcal{E}_{t}, t>0\right\}$ of complex separable Hilbert spaces satisfying $\mathcal{E}_{s} \otimes \mathcal{E}_{t} \cong \mathcal{E}_{s+t}$ in an associative way.

In precise terms we demand unitary operators $U_{s, t}: \mathcal{E}_{s} \otimes \mathcal{E}_{t} \rightarrow \mathcal{E}_{s+t}$ for $s, t>0$, with associative property:

$$
U_{s_{1}, s_{2}+s_{3}}\left(I \otimes U_{s_{2}, s_{3}}\right)=U_{s_{1}+s_{2}, s_{3}}\left(U_{s_{1}, s_{2}} \otimes I\right),
$$

for $s_{1}, s_{2}, s_{3}>0$. And here the measurability means the following:

$$
\mathcal{E}=\left\{(t, x): t \in(0, \infty), x \in \mathcal{E}_{t}\right\}
$$

is a standard Borel space, $\{t\} \times \mathcal{E}_{t}$ is a measurable subset of $\mathcal{E}$ and the maps

(i) $(x, y) \ni \mathcal{E}_{t} \times \mathcal{E}_{t} \rightarrow\langle x, y\rangle \ni \mathbb{C}$,

(ii) $((s, x),(t, y)) \ni \mathcal{E} \times \mathcal{E} \rightarrow\left(s+t, U_{s, t}(x, y)\right) \ni \mathcal{E}$

are measurable. Further one assumes that there exists a Borel isomorphism $\phi$ : $\mathcal{E} \rightarrow(0, t) \times \mathcal{M}$ for some fixed Hilbert space $\mathcal{M}$, such that $\phi$ restricted to fibre $\mathcal{E}_{t}$ is a unitary map onto $\{t\} \times \mathcal{M}$.

The last condition is equivalent to assuming that there exists a set $\left\{\left(e_{1}(t), e_{2}(t)\right.\right.$, $\ldots) ; t>0\}$, where for fixed $t,\left\{e_{1}(t), e_{2}(t), \ldots\right\}$ is an orthonormal basis for $\mathcal{E}_{t}$, and 
for fixed $n, t \rightarrow e_{n}(t)$ is a measurable map. The binary operation defined in (ii) is called a multiplication on $\mathcal{E}$. The notion of isomorphisms of product systems is the obvious one ([Ar1]). An anti-isomorphism is just like an isomorphism except that it reverses the order of multiplication. If $\mathcal{E}$ is a product system its opposite product system $\mathcal{E}^{*}$ is anti-isomorphic to $\mathcal{E}$ and is obtained through the reversed multiplication:

$$
((s, x),(t, y)) \rightarrow\left(s+t, U_{t, s}(y, x)\right) .
$$

Observe that in a product system $\mathcal{E}$, either $\operatorname{dim}\left(\mathcal{E}_{t}\right) \equiv 1$ or $\operatorname{dim}\left(\mathcal{E}_{t}\right) \equiv \aleph_{0}$. In the first case we say that the product system is trivial, and it turns out that up to isomorphisms there is only one trivial product system, the obvious one.

Now let us look at the family of Hilbert spaces $\left\{\mathcal{P}_{t}\right\}$ constructed in Section 4. We claim that

$$
\mathcal{P}=\left\{(t, \xi): t \in(0, \infty), \xi \in \mathcal{P}_{t}\right\} \subset(0, \infty) \times \mathcal{H},
$$

considered as a subset of $(0, \infty) \times \mathcal{H}$, is a product system with multiplication defined by

$$
((s, \xi),(t, \eta)) \rightarrow\left(s+t, U_{s, t}(\xi \otimes \eta)\right)
$$

for $(s, \xi),(t, \eta) \in \mathcal{P}$. Firstly we need to show that $\mathcal{P}$ is a Borel subset of $(0, \infty) \times \mathcal{H}$. ( $\mathcal{H}$ is a Hilbert space, so has its Borel $\sigma$-algebra coming from its natural topology.) This and the measurability of multiplication follows from the next proposition.

Proposition 5.5. (i) $\mathcal{P}$ is a closed subset of $(0, \infty) \times \mathcal{H}$.

(ii) The mapping $((s, \xi),(t, \eta)) \rightarrow\left(s+t, U_{s, t}(\xi \otimes \eta)\right)$ is jointly continuous on $\mathcal{P}$.

Proof. Suppose $\left(s_{n}, \xi_{n}\right) \in \mathcal{P}$ and converges to $(s, \xi)$ in $(0, \infty) \times \mathcal{H}$. Then

$$
j_{s_{n}}(|a\rangle\langle a|) \xi_{n}=\xi_{n} \text { for all } n
$$

and by strong continuity of $j, j_{s}(|a\rangle\langle a|) \xi=\xi$. This proves the first part. To prove (ii) consider a pair of sequences $\left(s_{n}, \xi_{n}\right),\left(t_{n}, \eta_{n}\right)$ converging to $(s, \xi),(t, \eta)$ in $\mathcal{P}$. We have

$$
\begin{aligned}
\left\|U_{s_{n}, t_{n}}\left(\xi_{n} \otimes \eta_{n}\right)-U_{s, t}(\xi \otimes \eta)\right\| & \leq\left\|U_{s_{n}, t_{n}}\left(\xi_{n} \otimes \eta_{n}\right)-U_{s, t_{n}}(\xi \otimes \eta)\right\| \\
& +\left\|U_{s, t_{n}}\left(\xi_{n} \otimes \eta_{n}\right)-U_{s, t}(\xi \otimes \eta)\right\| .
\end{aligned}
$$

Now note that $U_{s_{n}, t_{n}}\left(\xi_{n} \otimes \eta_{n}\right)=W_{s_{n}, t_{n}}\left(\xi_{n} \otimes \eta_{n}\right)=W_{\infty, t_{n}}\left(\xi_{n} \otimes \eta_{n}\right)$ from Remark 4.4. In a similar way, $U_{s, t_{n}}\left(\xi_{n} \otimes \eta_{n}\right)=W_{\infty, t_{n}}\left(\xi \otimes \eta_{n}\right)$. This helps to write the first term as $W_{\infty, t_{n}}\left(\left(\xi_{n}-\xi\right) \otimes \eta_{n}\right)$, which clearly converges to zero. As for the second term, use strong continuity of $j$ on vectors of the form $j(\underline{r}, \underline{Y}) u$ and then extend.

Proposition 5.6. There exists a Borel map $\phi: \mathcal{P} \rightarrow(0, \infty) \times \mathcal{K}$, for some Hilbert space $\mathcal{K}$ such that $\left.\phi\right|_{\{t\} \times \mathcal{K}}$ is a unitary map for every $t$.

Proof. Note that either $\operatorname{dim} \mathcal{P}_{t} \equiv \aleph_{0}$ or $\operatorname{dim} \mathcal{P}_{t} \equiv 1$ for $t>0$. The first case can be taken care of using Lemma 10.8.7 of [Di] (exactly as done by Arveson [Ar1]). If $\operatorname{dim} \mathcal{P}_{t} \equiv 1$, then we know that there exists a strongly continuous semigroup $U$ of unitaries (see remarks after Theorem 4.7) such that

$$
\theta_{t}(Z)=U_{t} Z U_{t}^{*}
$$

Now as $\mathcal{P}_{t}$ is the one dimensional space spanned by $U_{t} a$, it is clear that $\left(t, U_{t} a\right) \rightarrow$ $(t, 1)$ defines a Borel mapping of $\mathcal{P}$ to $(0, \infty) \times \mathbb{C}$. 
Theorem 5.7. Consider a unitary path-continuous quantum dynamical semigroup. Then the space $\mathcal{P}$ associated with it is a continuous tensor product system of Hilbert spaces.

Proof. This claim is a summary of (4.7) and Propositions 5.4- 5.6.

\section{ON INDEX}

The notion of index for $E_{0}$-semigroups was introduced by Powers. Powers and Robinson described as to when we can say two semigroups have the same index. A clear-cut definition of index was given by Arveson with

$$
\operatorname{index}(\alpha)=[\mathcal{E}],
$$

that is, index is the isomorphism class of the product system $\mathcal{E}$ associated with the semigroup as in [Ar1]. Now given a quantum dynamical semigroup (pathcontinuous, conservative) $T$ on $\mathcal{B}\left(\mathcal{H}_{0}\right)$, it is natural to define its index by

$$
\operatorname{index}(T)=\operatorname{index}(\theta)
$$

where $\theta$ is the associated semigroup of endomorphisms (see Theorem 4.7). We are not interpreting this notion of index here. The interested reader may see [Ar3], where it is compared with Fredholm index of isometries or co-isometries. It has also been pointed out that the analogy does not go very far. The reason could be that isometries or semigroups of isometries are more connected with the 'additive structure' $\mathcal{N}$, and have very little to do with the product systems (see comments after Theorem 4.8). In any case, it should be pretty clear, even by looking at discrete time case, that the index is an important invariant for the semigroup.

It turns out that index $(T)=\left[\mathcal{P}^{*}\right], \mathcal{P}^{*}$ being the opposite product system of $\mathcal{P}$. To see this we outline Arveson's construction of product systems based on

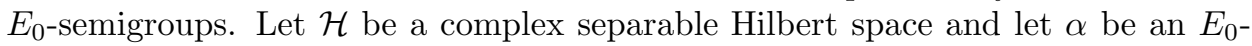
semigroup on $\mathcal{B}(\mathcal{H})$. Take

$$
E_{t}=\left\{Y \in \mathcal{B}(\mathcal{H}): \alpha_{t}(X) Y=Y X\right\}
$$

that is, $Y$ intertwines between $\alpha_{t}$ and identity representation of $\mathcal{B}(\mathcal{H})$. For $Y, Z$ in $E_{t}$, the commutation relation in the definition of $E_{t}$ implies that $Y^{*} Z$ commutes with every $X \in \mathcal{B}(\mathcal{H})$. Hence $Y^{*} Z$ is a scalar. Define $\langle Y, Z\rangle$ by putting $Y^{*} Z=$ $\langle Y, Z\rangle$. Then it can be shown that $E_{t}$ becomes a separable Hilbert space with this innerproduct. The tensor product structure on the family $\left\{E_{t}\right\}$ is just operator multiplication. Observe that if $Y \in E_{s}, Z \in E_{t}$, then $Y Z$ is in $E_{s+t}$. Now it is a routine matter to check that $U_{s, t}: E_{s} \otimes E_{t} \rightarrow E_{s+t}$, defined by

$$
U_{s, t}(Y \otimes Z)=Y Z
$$

is a unitary operator and

$$
\mathcal{E}=\left\{(t, Y): t \in(0, \infty), Y \in E_{t}\right\}
$$

considered as a measurable subset of $(0, \infty) \times \mathcal{B}(\mathcal{H})$ (with strong topology on $\mathcal{B}(\mathcal{H})$ ) is a product system.

We shall call $\mathcal{E}$ the canonical product system associated with the $E_{0}$-semigroup $\alpha$. From the penetrating study of Arveson [Ar6] one knows that every product system arises this way.

Theorem 6.1. The canonical product system $\mathcal{E}$ associated with the $E_{0}$-semigroup $\theta$ (of Theorem 4.7) is anti-isomorphic to the product system $\mathcal{P}$. 
B. V. RAJARAMA BHAT

Proof. First observe that if $Z \in \mathcal{E}_{t}$ then $Z a \in \mathcal{P}_{t}$ as

$$
\theta_{t}(|a\rangle\langle a|) Z a=Z|a\rangle\langle a| a=Z a .
$$

Now consider the map $\psi: \mathcal{E} \rightarrow \mathcal{P}$, defined by

$$
\psi(s, Z)=(s, Z a) .
$$

It is easy to see that $\psi$ is an isometry on each fibre $\mathcal{E}_{t}$. Further for $\xi \in \mathcal{P}_{s}$, consider the bounded operator $Z_{\xi}$ on $\mathcal{H}$ defined by

$$
Z_{\xi} \gamma=\theta_{s}(|\gamma\rangle\langle a|) \xi, \quad \gamma \in \mathcal{H} .
$$

For $W \in \mathcal{B}(\mathcal{H})$ and $\gamma \in \mathcal{H}$, we have

$$
\theta_{s}(W) Z_{\xi} \gamma=\theta_{s}(W) \theta_{s}(|\gamma\rangle\langle a|) \xi=\theta_{s}(|W \gamma\rangle\langle a|) \xi=Z_{\xi} W \gamma .
$$

That is, $Z_{\xi} \in \mathcal{E}_{s}$. Further $Z_{\xi} a=\theta_{s}(|a\rangle\langle a|) \xi=\xi$, and hence $\psi$ is surjective. It is obvious that $\psi$ is a Borel map. Now consider $\xi \in \mathcal{P}_{s}, \eta \in \mathcal{P}_{t}$ with associated $Z_{\xi}$, $Z_{\eta}$. Clearly

$$
\begin{aligned}
U_{s, t}(\xi \otimes \eta) & =U_{s, t}\left(\theta_{s}(|a\rangle\langle a|) \xi \otimes \theta_{t}(|a\rangle\langle a|) \eta\right) \\
& =\theta_{s+t}(|a\rangle\langle a|) \theta_{t}(|\xi\rangle\langle a|) \eta \\
& =\theta_{t}\left(\left|\theta_{s}(|a\rangle\langle a|) \xi\right\rangle\langle a|\right) \eta \\
& =Z_{\eta} Z_{\xi} a .
\end{aligned}
$$

Note that the order of $\xi, \eta$ gets reversed. And hence $\psi$ is an anti-isomorphism.

There is nothing deep in getting an ant-isomorphism and not an isomorphism here. One can incorporate a somewhat unnatural twist in the definition of $W_{s, t}, U_{s, t}$ to obtain an isomorphism above. Such a definition would also have the undesirable effect of shifting the initial space $\mathcal{H}_{0}$ into the second component in the factorization of $j$.

The index enjoys some natural functorial properties. A semigroup $S$ acting on $\mathcal{B}\left(\mathcal{K}_{0}\right)$ is said to be conjugate to $T$ if there exists a unitary map $V: \mathcal{H}_{0} \rightarrow \mathcal{K}_{0}$ such that

$$
S_{t}(X)=V T_{t}\left(V^{*} X V\right) V^{*}, \text { for } X \in \mathcal{K}_{0}, t \geq 0 .
$$

It is obvious that if $T, S$ are conjugate then they have the same index. Now suppose $T, R$ are quantum dynamical semigroups both acting on $\mathcal{B}\left(\mathcal{H}_{0}\right)$. We say that $T, R$ are exterior equivalent if there exists a strongly continuous family of unitary operators $\left\{U_{t}\right\}$ on $\mathcal{B}\left(\mathcal{H}_{0}\right)$ satisfying

$$
T_{t}(X)=U_{t}^{*} R_{t}(X) U_{t},
$$

for $X \in \mathcal{B}\left(\mathcal{H}_{0}\right), t \geq 0$. Following the footsteps of Arveson we have the notion of cocycle conjugacy (In $[$ Ar1] this was called outer conjugacy.)

Definition 6.2. Let $T$ (resp. $S$ ) be a quantum dynamical semigroup acting on $\mathcal{B}\left(\mathcal{H}_{0}\right)$ (resp. $\mathcal{B}\left(\mathcal{K}_{0}\right)$ ). Then $T$ and $S$ are said to be cocycle conjugate if there exists a third semigroup $R$ acting on $\mathcal{B}\left(\mathcal{H}_{0}\right)$ conjugate to $S$ and exterior equivalent to $T$.

Theorem 6.3. If two quantum dynamical semigroups are cocycle conjugate then they have the same index. 
Proof. Clearly it is enough to show that exterior equivalent semigroups have the same index. So let $T, R$ be semigroups acting on $\mathcal{B}\left(\mathcal{H}_{0}\right)$, with a family of unitary operators $\left\{U_{t}\right\}$, satisfying (6.1). We obtain an isomorphism between product systems $\mathcal{P}_{T}$ and $\mathcal{P}_{R}$. Let $(\mathcal{H}, F, j),\left(\mathcal{H}^{\prime}, F^{\prime}, j^{\prime}\right)$ be minimal dilations of $T, R$ respectively. Note that for $(\underline{r}, \underline{Y}, u),(\underline{r}, \underline{Z}, v) \in \mathcal{D}$ (take $X=I$ in $(2.6)$ )

$$
\begin{aligned}
\langle j(\underline{r}, \underline{Y}) u, j(\underline{r}, \underline{Z}) v\rangle & \\
& =\left\langle u, T_{r_{n}}\left(Y_{n}^{*} T_{r_{n-1}-r_{n}}\left(Y_{n-1}^{*} \cdots Y_{2}^{*} T_{r_{1}-r_{2}}\left(Y_{1}^{*} Z_{1}\right) Z_{2} \cdots Z_{n-1}\right) Z_{n}\right) v\right\rangle \\
& =\left\langle u, U_{r_{n}}^{*} R_{r_{n}}\left(Y_{n}^{*} \cdots Y_{2}^{*} U_{r_{1}-r_{2}}^{*} R_{r_{1}-r_{2}}\left(Y_{1}^{*} Z_{1}\right) U_{r_{1}-r_{2}} Z_{2} \cdots Z_{n}\right) U_{r_{n}} v\right\rangle .
\end{aligned}
$$

This shows that the map $L: \mathcal{H} \rightarrow \mathcal{H}^{\prime}$ defined by

$$
L j(\underline{r}, \underline{Y}) u=j^{\prime}\left(\underline{r},\left(Y_{1}, U_{r_{1}-r_{2}} Y_{2}, \ldots, U_{r_{n-1}-r_{n}} Y_{n}\right) U_{r_{n}} u\right.
$$

is an isometry. Now as $\underline{Y}$ was arbitrary in $(\underline{r}, \underline{Y}, u)$, the range of $L$ is total and hence $L$ is a unitary operator. Observe that $L$ maps range of $j_{t}(|a\rangle\langle a|)$ to the range of $j_{t}^{\prime}(|a\rangle\langle a|)$. Now it is not hard to see that the mapping $(t, \xi) \rightarrow(t, L \xi)$ is an isomorphism between product systems $\mathcal{P}_{T}$ and $\mathcal{P}_{R}$.

It may be noted that for $E_{0}$-semigroups the converse of Theorem 6.3 is also true. That is, if two $E_{0}$-semigroups have same index then they are cocycle conjugate. But clearly this no longer holds in the general situation as that would mean that every quantum dynamical semigroup is cocycle conjugate to some $E_{0}$-semigroup.

Observe that it is meaningful to talk of tensor products of product systems (see [Ar1], pp. 29). Suppose T, $S$ are quantum dynamical semigroups on $\mathcal{B}\left(\mathcal{H}_{0}\right), \mathcal{B}\left(\mathcal{K}_{0}\right)$ with respective minimal dilations $(\mathcal{H}, F, j),(\mathcal{K}, G, k)$. It is not difficult to see that $(\mathcal{H} \otimes \mathcal{K}, F \otimes G, j \otimes k)$ defined by $(F \otimes G)(t)=F(t) \otimes G(t),(j \otimes k)_{t}=j_{t} \otimes k_{t}$, is a minimal dilation of the semigroup $T \otimes S$. We also see that $\theta_{T \otimes S}=\theta_{T} \otimes \theta_{S}$. Now it follows that

$$
\operatorname{index}(T \otimes S)=\operatorname{index}\left(\theta_{T}\right) \otimes\left(\theta_{S}\right)=\left[\mathcal{P}_{T}^{*} \otimes \mathcal{P}_{S}^{*}\right] .
$$

The problem of determining the index for different quantum dynamical semigroups remains open. Looking at the well-developed theory of Evans-Hudson flows ([Me], $[\mathrm{Pa}]$ ) one feels that perhaps the index of nice (say of bounded generator) quantum dynamical semigroups are exponential (Fock) product systems and can be obtained through the theory of quantum stochastic differential equations. This we have been able to verify only for some very special examples [BF].

Recall that by a unit of a product system $\mathcal{P}$ one means a non-zero measurable cross section $t \in(0, \infty) \rightarrow u_{t} \in \mathcal{P}_{t}$, such that

$$
u(s+t)=U_{s, t}\left(u_{s} \otimes u_{t}\right), \quad s, t>0 .
$$

The numerical index for the semigroup is the dimension of a Hilbert space constructed out of units (see [Ar1]) of the associated product system. So units play a very crucial role in understanding product systems. The following theorem provides some units through completely elementary means in some special cases. As before we are considering a path-continuous dynamical semigroup $T$ acting on $\mathcal{B}\left(\mathcal{H}_{0}\right)$, with associated product system $\mathcal{P}$ defined using a unit vector $a \in \mathcal{H}_{0}$.

Theorem 6.4. If $b$ is a unit vector in $\mathcal{H}_{0}$ satisfying

$$
T_{t}(|b\rangle\langle b|) b=e^{-q t} b, \quad t \geq 0,
$$

for some $q \geq 0$, then the cross-section $u$ defined by $u_{t}=j_{t}(|a\rangle\langle b|) b$ is a unit of $\mathcal{P}$. 
Proof. Clearly $u_{t} \in \mathcal{P}_{t}$, and the mapping $t \rightarrow u_{t}$ is continuous (and hence measurable). Now

$$
\begin{aligned}
U_{s, t}\left(u_{s} \otimes u_{t}\right) & =U_{s, t}\left(j_{s}(|a\rangle\langle b| b) \otimes j_{t}(|a\rangle\langle b|) b\right) \\
& =j_{s+t}(|a\rangle\langle b|) j_{t}(|b\rangle\langle b|) b,
\end{aligned}
$$

and on using (4.14) we obtain

$$
\begin{aligned}
\left\|j_{s+t}(|a\rangle\langle b|) j_{t}(|b\rangle\langle b|) b\right\|^{2} & =\left\langle b, T_{t}\left(|b\rangle\left\langle b\left|T_{s}(|b\rangle\langle a|| a\rangle\langle b|)\right| b\right\rangle\langle b|\right) b\right\rangle \\
& =\left\langle b, T_{t}(|b\rangle\langle b|)\right\rangle \cdot\left\langle b, T_{s}(|b\rangle\langle b|) b\right\rangle \\
& =e^{-q(s+t)} .
\end{aligned}
$$

Similar computations of other terms involved yields $\left\|U_{s, t}\left(u_{s} \otimes u_{t}\right)-u_{s+t}\right\|^{2}=0$.

The special case of $q=0$ in Theorem 6.4 might be worth noting, where we are demanding a pure invariant state for $T$. Semigroups with such states appear in various contexts. See [Da4] for a study of their generators.

\section{ACKNOWLEDGEMENTS}

Most of this work was done while I was enjoying the generous hospitality of F. Fagnola at Pisa. Earlier I had some useful discussions with K.R. Parthasarathy and K.B. Sinha at ISI, New Delhi. I also would like to thank W. Arveson for sending me a bunch of his reprints on my request, which helped me to borrow from many of his ideas.

\section{REFERENCES}

[AFL] Accardi, L., Frigerio, A., Lewis, J.T. : Quantum Stochastic Processes, Publ. Res. Inst. Math. Sci., 18 (1982), 97-133. MR 84m:82031

[AL] Alicki, R., Lendi, K. : Quantum Dynamical Semigroups and Applications, Springer Lecture Notes in Phys. 286 (1987), Berlin. MR 89b:81002

[Ar1] Arveson, W. : Continuous Analogues of Fock Space, Mem. Amer. Math. Soc., Vol. 80, No.409 (1989). MR f:47061

[Ar2] - An addition formula for the index of semigroups of endomorphisms of $\mathcal{B}(H)$, Pacific J. Math., 137 (1989), 19-36. MR 90c:47074

[Ar3] : The spectral $C^{*}$-algebra of an $E_{0}$-semigroup, Proc. of Symposia on Pure Math., 51 (1990), 1-15. MR 92c:46074

[Ar4] _ Continuous analogues of Fock space II : the spectral $C^{*}$-algebra, J. Funct. Anal., 90 (1990), 138-205. MR 91d:46073

[Ar5] _ Continuous analogues of Fock space III : singular states, J. Operator Theory, 22 (1989), 165-205. MR 90m:46118

[Ar6] C Continuous analogues of Fock space IV : essential states, Acta Math., 164 (1990), 265-300. MR 91d:46074

[Ar7] _ : $C^{*}$-algebras associated with sets of semigroups of isometries, International J. of Math. 2 (1991), 235-255. MR 92i:46082

[AW] Araki, H., Woods, E.J.: Complete Boolean algebras of type I factors, Publ. Res. Inst. Math. Sci., Series A, Vol II (1966) 157-242. MR 35:748

[BF] Bhat, B.V.R., Fagnola F. : On minimality of Evans-Hudson flows, (preprint).

[Bh] Bhat, B.V.R. : Markov Dilations of Nonconservative Quantum Dynamical Semigroups and a Quantum Boundary Theory, Ph. D. Thesis, Indian Statistical Institute, New Delhi (1993).

[BP2] Bhat, B.V. R., Parthasarathy, K.R. : Markov dilations of nonconservative dynamical semigroups and a quantum boundary theory, Ann. Inst. H. Poincaré Probab. Statist., (to appear).

[BP3] Bhat, B.V. R., Parthsarathy, K.R. : Kolmogorov's existence theorem for Markov processes in $C^{*}$ algebras, Proc. Ind. Acad. Sci. Math. Sci., 103 (1994), 253-262. CMP 94:13 
[Da1] Davies, E.B. : Quantum Theory of Open Systems, Academic Press (1976) New York. MR $\mathbf{5 8 :} 8853$

[Da2] Davies, E.B. : Quantum dynamical semigroups and the neutron diffusion equation, Rep. Math. Phys. 11 (1977), 169-189. MR 58:20111

[Da3] Davies, E.B. : One-Parameter Semigroups, Academic Press (1980), New York. MR 82i: 47060

[Da4] Davies, E.B. : Generators of dynamical semigroups, J. Funct. Anal. 34 (1979), 421-432. MR 81a:47039

[Di] Dixmier, J. : Les $C^{*}$-algèbres et leurs représentations, Gauthier-Villars (1964) Paris. MR 80:1404

[EL] Evans, D.E., Lewis, J.T. : Dilations of Irreversible Evolutions in Algebraic Quantum Theory, Comm. Dublin Inst. Adv. Stud., Ser. A no.24 (1977). MR 58:8915

[Em] Emch, G. G. : Minimal dilations of CP flows, $C^{*}$ algebras and applications to physics, Springer Lecture Notes in Math. 650 (1978), 156-159. MR 81a:46079

[GKS] Gorini, V., Kossakowski, A., Sudarshan, E.C.G. : Completely positive dynamical semigroups of $n$-level systems, J. Math. Phys. 17 (1976), 821-825. MR 53:9998

[Gu] Guichardet, A.: Symmetric Hilbert spaces and related topics, Springer Lecture Notes in Math. 261 (1972) Berlin. MR 58:12416

[HP] Hudson, R.L. Parthasarathy, K.R. : Stochastic dilations of uniformly continuous completely positive semigroups, Acta Appl. Mathematicae 2 (1984), 353-398. MR 85i:46092

[Li] Lindblad, G. : On the generators of quantum dynamical semigroups, Comm. Math. Phys. 48 (1976), 119-130. MR 54:1990

[Me] Meyer, P.A. : Quantum Probability for Probabilists, Springer Lecture Notes in Math. 1538, (1993) Berlin. MR 94k:81152

[Pa] Parthasarathy, K.R. : An Introduction to Quantum Stochastic Calculus, Monographs in Math., Birkhäuser Verlag (1992) Basil. MR 93g:81062

[PaS] Parthasarathy, K.R., Schmidt, K. : Positive Definite Kernels, Continuous Tensor Products and Central Limit Theorems of Probability Theory, Springer Lecture Notes in Math. 272 (1972) Berlin. MR 58:29849

[Po1] Powers, R.T. : An index theory for semigroups of endomorphisms of $\mathcal{B}(H)$ and type $I I_{1}$ factors, Canad. J. Math., 40 (1988), 86-114. MR 89f:46116

[Po2] Powers, R. T. : A non-spatial continuous semigroup of $*$-endomorphisms of $\mathcal{B}(H)$, Publ. Res. Inst. Math. Sci. 23 (1987), 1053-1069. MR 89f:46118

[PP] Powers, R.T., Price, G. : Continuous spatial semigroups of $*$-endomorphisms of $\mathcal{B}(\mathcal{H})$, Trans. Amer. Math. Soc., 321 (1990), 347-361. MR 90m:47058

[PR] Powers, R.T., Robinson D. : An index for continuous semigroups of $*$ endomorphisms of $\mathcal{B}(H)$, J. Funct. Anal., 84 (1989), 85-96. MR 90f: 46107

[Pr] Price, G. : Shifts on type $I I_{1}$ factors, Canad. J. Math., 39 (1987), 492-511. MR 88i:46080

[Sa] Sauvageot, J-L. : Markov quantum semigroups admit covariant Markov $C^{*}$ dilations, Comm. Math. Phys. 106 (1986), 91-103. MR 88a:46077

[St] Stinespring, W.F. : Positive functions on $C^{*}$ algebras, Proc. Amer. Math Soc. 6 (1955), 211-216. MR 16:1033b

[SzF] Sz.-Nagy, B., Foias, C. : Harmonic Analysis of Operators on Hilbert Space, North-Holland (1970) Amsterdam. MR 37:778

[Va] Varadarajan, V.S. : Geometry of Quantum Theory, Second Edition, Springer (1985) Berlin. MR 87a:81009

[ViS] Vincent-Smith, G. F. : Dilations of a dissipative quantum dynamical system to a quantum Markov process, Proc. London Math. Soc.(3), 49 (1984), 58-72. MR 85i:46086

The Fields Institute, 222 College Street, Toronto, Ontario, Canada

E-mail address: bhat@fields.utoronto.ca

E-mail address: bhat@math.toronto.edu 